\title{
Convergence Rates of Multiscale and Wavelet Expansions
}

\author{
Mark A. Kon ${ }^{1}$ \\ Boston University and University of Warsaw \\ Louise Arakelian Raphael ${ }^{2}$ \\ Howard University
}

\begin{abstract}
Several results are proved which characterize the rate at which wavelet and multiresolution expansions converge to functions in a given Sobolev space in the supremum error norm. Some of the results are proved without assuming existence of a scaling function in the multiresolution analysis. Necessary and sufficient conditions are given for convergence at given rates in terms of behavior of Fourier transforms of the wavelet or scaling function near the origin. Such conditions turn out in special cases to be equivalent to moment conditions and other known conditions determining convergence rates.
\end{abstract}

\footnotetext{
${ }^{1}$ Research partially supported by the Air Force Office of Scientific Research, the National Science Foundation, and the U.S. Fulbright Commission

${ }^{2}$ Research partially supported by the Air Force Office of Scientific Research and the National Security Agency.
}

1991 AMS Mathematics Subject Classifications - Primary 42C15; Secondary 40A30 


\section{Introduction and definitions}

The computational efficiency of wavelet expansions is related to their multiresolution form and other well-studied properties. Wavelets are local in time and frequency, and a wavelet basis for $L^{2}\left(\mathbb{R}^{d}\right)$ consists of translations and dilations of one or more functions. Given a multiresolution expansion, it is natural to ask how fast the worst error of such an expansion decreases when applied to functions in a given class, or equivalently how fast the associated error operators converge to zero.

In this paper we state a number of equivalent conditions for given pointwise (sup norm) convergence rates of expansions of functions in Sobolev spaces, stated in terms of the wavelet, scaling function, symbol of scaling function, and projection operators associated with the multiresolution analysis (MRA). Our proofs expolit two facts. The first is that convergence rates of error operators for multiresolution analyses in given normed spaces are more natural to study using so-called associated homogeneous spaces, because of their better scaling properties. The second is that under assumptions more general than $\mathrm{r}$ regularity, the reproducing kernel associated with a projection operator is bounded by an $L^{1}$ radially decreasing convolution kernel. We assume only that the scaling function or weighted wavelets are bounded by an $L^{1}$ radial decreasing function. We emphasize that our convergence rates for projection operators continue to hold if we do not assume existence of a scaling functions or wavelets for the MRA

The present results apply to multiscale expansions including those using Haar and Daubechies wavelets, nonorthogonal wavelet expansions, and best approximations using spline functions. Our results hold for our spaces maximally, i.e., they do not generically hold for larger spaces of wavelets or the functions being expanded, if either one or the other is fixed. Some of the results in this paper have been announced previously [KKR2, KR2]. Operating under minimal hypotheses, we provide proofs here of results whose proofs were omitted or tersely sketched in the above announcements. While this paper is self-contained, we refer the reader to [KKR2, KR2] for preliminary results not included here.

Our results differ somewhat from Jackson-type theorems in Fourier analysis and characterizations of function spaces by convergence rates of their wavelet expansions (e.g., [Ma, Me]). In Fourier analysis convergence rates are determined by smoothness of functions being expanded. In wavelet expansions, rates of convergence are jointly determined by smoothness of the expanded function $f$, and characteristics of the wavelet (or scaling function). We attempt to pin down this dependence, focusing in this paper on limitations imposed by the wavelet or scaling function itself. This falls closer to the intent of the Strang-Fix type results of approximation theory [SF] in focusing on properties of the approximating function $\phi$ as well as smoothness of $f$. There $\phi$ must satisfy specific conditions for given approximation orders even if $f$ has infinite smoothness and compact support. It is shown in [Wa] that wavelet expansions of sufficiently smooth functions converge at rates commensurate with the differentiability of the wavelet. Such results are sharpened here into necessary and sufficient form, in $d$ dimensions and for larger classes of scaling functions and wavelets. Finally, we note results exist which provide $L^{2}$ [BDR, JZ] and supremum norm error bounds for shift-invariant spaces.

A classical multiresolution analysis (MRA; [Ma], [Me], [D2]) is a decomposition of $L^{2}\left(\mathbb{R}^{d}\right)$ into a sequence of closed subspaces $\mathrm{V}_{\mathrm{n}}$, 


$$
\ldots V_{-2} \subset V_{-1} \subset V_{0} \subset V_{1} \subset V_{2} \ldots,
$$

such that

$$
\phi(x) \in V_{i} \text { if and only if } \phi(2 x) \in V_{i+1} \text { for all } i .
$$

In addition it is assumed

$$
\begin{gathered}
\bigcap_{j \in \mathbb{Z}} V_{j}=\{0\} ; \\
\bigcup_{j \in \mathbb{Z}} V_{j}=L^{2}\left(\mathbb{R}^{d}\right)
\end{gathered}
$$

where overline denotes closure; and $\mathrm{V}_{0}$ is closed under integer translations,

$$
\phi(x) \in V_{0} \quad \Rightarrow \quad \phi(x-k) \in V_{0},
$$

for $\mathrm{k} \in \mathbb{Z}^{\mathrm{d}}$.

Finally, it is usually assumed in an MRA (though we do not assume this a priori) that there exists $\phi \in \mathrm{L}^{2}\left(\mathbb{R}^{\mathrm{d}}\right)$ such that

$$
\left\{\phi_{k}(x) \equiv \phi(x-k)\right\}_{k \in \mathbb{Z}^{d}} \text { form an orthonormal basis for } V_{0} .
$$

Such a $\phi$ is a scaling function. Our convention is that a multiresolution analysis is a sequence of orthogonal projections $\left\{P_{n}\right\}$ onto the above spaces $\left\{V_{n}\right\}$ which satisfies (1.1ad), and existence of $\phi$ will be assumed only when specified.

Let $\mathrm{W}_{\mathrm{i}}$ be the orthocomplement of $\mathrm{V}_{\mathrm{i}}$ in $\mathrm{V}_{\mathrm{i}+1}$, i.e., $\mathrm{W}_{\mathrm{i}}=\mathrm{V}_{\mathrm{i}+1} \ominus \mathrm{V}_{\mathrm{i}}$, so $\mathrm{V}_{\mathrm{i}+1}=\mathrm{V}_{\mathrm{i}} \oplus \mathrm{W}_{\mathrm{i}}$. From existence of $\phi$ it follows (see, e.g., [D2]) that there are basic wavelets $\left\{\psi^{\lambda}(\mathrm{x})\right\}_{\lambda \in \Lambda}$ (with $\Lambda$ finite) such that $\psi_{\mathrm{jk}}^{\lambda}(\mathrm{x}) \equiv 2^{\mathrm{j} d / 2} \psi^{\lambda}\left(2^{\mathrm{j}} \mathrm{x}-\mathrm{k}\right) \quad\left(\mathrm{j} \in \mathbb{Z}, \mathrm{k} \in \mathbb{Z}^{\mathrm{d}}\right)$ form an orthonormal basis for a fixed $\mathrm{W}_{\mathrm{j}}$, and form an orthonormal basis for $\mathrm{L}^{2}\left(\mathbb{R}^{\mathrm{d}}\right)$ as $\mathrm{j}$, $\mathrm{k}$ vary.

By (1.1a) and (1.1b), there exist $\left\{\mathrm{h}_{\mathrm{k}}\right\}_{k \in \mathbb{Z}^{d}}$ such that

$$
\phi(x)=2^{d} \sum_{k \in \mathbb{Z}^{d}} h_{k} \phi(2 x-k) .
$$

The function

$$
m_{0}(\xi) \equiv \sum_{k \in \mathbb{Z}^{d}} h_{k} e^{-i k \cdot \xi},
$$

is the symbol of $\left\{\mathrm{h}_{\mathrm{k}}\right\}_{\mathrm{k} \in \mathbb{Z}^{\mathrm{d}}}$, and is easily shown to satisfy

$$
\hat{\phi}(\xi)=m_{0}(\xi / 2) \hat{\phi}(\xi / 2) .
$$

Our convention for Fourier transforms is

$$
\widehat{\phi} \equiv \mathcal{F}(\phi) \equiv(2 \pi)^{-d / 2} \int_{\mathbb{R}^{d}} \phi(x) e^{-i \xi \cdot x} d x
$$


we will write $\xi \cdot x=\xi x$.

The most direct construction of multidimensional wavelets is through tensor products of one dimensional multiresolution analyses (see, e.g., [D2]). In general we write, as a wavelet basis for $\mathrm{L}^{2}\left(\mathbb{R}^{\mathrm{d}}\right)$, the collection $\left\{\psi_{\mathrm{jk}}^{\lambda}\right\}_{\mathrm{j} \in \mathbb{Z}, \mathrm{k} \in \mathbb{Z}^{\mathrm{d}}, \lambda \in \Lambda}$, with $\Lambda$ an indexing set containing $2^{\mathrm{d}}-1$ elements.

A general construction of so-called $r$-regular wavelet bases in multiple dimension is given in [Gr, Me,Wo], where the scaling function $\phi$ is assumed r-regular. Note that in this algorithm, the assumption of r-regularity may be weakened somewhat, in particular with respect to the requirements of rapid decay at infinity for the scaling function $\phi$.

Whichever construction is used (see [D2], Ch.10; [Me], [Gr]), our results hold for any set $\left\{\psi^{\lambda}\right\}_{\lambda}$ whose translations and dilations form an orthonormal basis for $\mathrm{L}^{2}\left(\mathbb{R}^{\mathrm{d}}\right)$. We will assume our wavelets $\psi^{\lambda}$ and scaling functions $\phi$ are in $L^{2}$, and that they are radially bounded by decreasing $L^{1}$ functions (the details are given below).

Definitions 1.1: A function $\mathrm{f}(\mathrm{x})$ on $\mathbb{R}^{\mathrm{d}}$ is radial if $\mathrm{f}$ depends on $|\mathrm{x}|$. A real radial function is decreasing if $\mathrm{f}(\mathrm{x}) \leq \mathrm{f}(\mathrm{y})$ whenever $|\mathrm{x}| \geq|\mathrm{y}|$. A function $\mathrm{f}(\mathrm{x})$ is in the class [RB] (c.f. [GK1,2]) if it is absolutely bounded by an $\mathrm{L}^{1}$ radial decreasing function $\eta(\mathrm{x})$, and $\eta \in \mathrm{L}^{1}\left(\mathbb{R}^{\mathrm{d}}\right)$. (Note since $\eta$ is defined at the origin, $\phi$ must be bounded).

Let $\mathrm{P}_{\mathrm{n}}$ and $\mathrm{Q}_{\mathrm{n}}$, respectively, be the orthogonal projections from $L^{2}\left(\mathbb{R}^{d}\right)$ onto $\mathrm{V}_{\mathrm{n}}$ and $\mathrm{W}_{\mathrm{n}}$, with kernels (when they exist) $\mathrm{P}_{\mathrm{n}}(\mathrm{x}, \mathrm{y})$ and $\mathrm{Q}_{\mathrm{n}}(\mathrm{x}, \mathrm{y})$. Define $P=P_{0}$. An operator $T$ is in [RB] if it has a kernel $|T(x, y)| \leq K(x-y)$, with $K(\cdot) \in[\mathrm{RB}]$.

Given $\mathrm{f} \in \mathrm{L}^{2}$,

(i) the multiresolution approximation of $\mathrm{f}$ is defined by the sequence $\left\{\mathrm{P}_{\mathrm{n}} \mathrm{f}\right\}_{\mathrm{n}}$.

(ii) the wavelet expansion of $\mathrm{f}$ is

$$
\sum_{j ; k ; \lambda} a_{j k}^{\lambda} \psi_{j k}^{\lambda}(x) \sim f
$$

with $\mathrm{a}_{\mathrm{jk}}^{\lambda}$ the $\mathrm{L}^{2}$ expansion coefficients of $f$, where $\sim$ denotes convergence in $L^{2}$.

(iii) the scaling expansion of $\mathrm{f}$ is

$$
\sum_{k} b_{k} \phi(x)+\sum_{j \geq 0 ; k ; \lambda} a_{j k}^{\lambda} \psi_{j k}^{\lambda}(x) \sim f
$$

where the $b_{k}, a_{j k}^{\lambda}$ are $L^{2}$ expansion coefficients of $f$. We say that such sums converge in a given sense (e.g., pointwise, in $\mathrm{L}^{\mathrm{p}}$, etc.), if they do so in a semi-order-independent way. Specifically our convention is that sums are calculated such that the range (largest minus smallest) of $\mathrm{j}$ values for which the sum over $\mathrm{k}, \lambda$ is incomplete remains uniformly bounded.

Finally we define a scaling function of the MRA $\left\{P_{n}\right\}$ to be any function $\phi$ whose integer translates generate the space $V_{0}$, the range of $P_{0}$ (we do not assume existence of a $\phi$ a priori for an MRA). Since $\phi$ uniquely determines a symbol $m_{0}(\xi)$ (equation (1.3)), we define any symbol arising from a $\phi$ corresponding to $\left\{P_{n}\right\}$ to be a symbol of $\left\{P_{n}\right\}$.

Our results on sup-norm convergence norm extend a body of results (e.g., see [Wa, $\mathrm{Ma}, \mathrm{Me}, \mathrm{SP}, \mathrm{BR}, \mathrm{JZ}])$. Mallat [Ma] was the first to sharply measure decay in $n$ of $\mathrm{L}^{2}$ 
approximation error, i.e., $\left\|\mathrm{f}-\mathrm{P}_{\mathrm{n}} \mathrm{f}\right\|_{\mathrm{L}^{2}}$ for $r$-regular wavelets, resulting in a characterization of Sobolev spaces in terms of error decay rates of wavelet series. Our results measure $L^{\infty}$ error, yielding best possible conditions allowing given convergence rates.

Definition 1.2: The Sobolev space of order $s$ is

$$
\mathrm{H}^{\mathrm{s}} \equiv\left\{f \in \mathrm{L}^{2}\left(\mathbb{R}^{\mathrm{d}}\right):\|\mathrm{f}\|_{\mathrm{s}} \equiv \sqrt{\int|\hat{\mathrm{f}}(\xi)|^{2}\left(1+|\xi|^{2}\right)^{\mathrm{s}} \mathrm{d} \xi}<\infty\right\} .
$$

The homogeneous Sobolev space is:

$$
\mathrm{H}_{\mathrm{h}}^{\mathrm{s}} \equiv\left\{f \in \mathrm{L}^{2}\left(\mathbb{R}^{\mathrm{d}}\right):\|\mathrm{f}\|_{\mathrm{h}, \mathrm{s}} \equiv \sqrt{\int|\hat{\mathrm{f}}(\xi)|^{2}|\xi|^{2 \mathrm{~s}} \mathrm{~d} \xi}<\infty\right\}
$$

Note $\mathrm{H}_{\mathrm{h}}^{\mathrm{s}}$ is incomplete in this norm since it is restricted to $L^{2}\left(\mathbb{R}^{d}\right)$. In Fourier space, it is a dense subspace of the complete weighted $\mathrm{L}^{2}$ space of all measurable $\hat{\mathrm{f}}$ with $\|\mathrm{f}\|_{\mathrm{h}, \mathrm{s}}<\infty$. This space is advantageous in that its norms change simply with of scale. By our restriction that the functions be in $L^{2}$, in fact for $s \geq 0, H^{s}$ and $H_{h}^{s}$ consist of the same $L^{2}$ functions, with quite different norms.

Definition 1.3: An (MRA) $\left\{P_{n}\right\}$ or wavelet family $\psi^{\lambda}$ yields pointwise order of approximation (or convergence) $\mathrm{s}$ in $\mathrm{H}^{\mathrm{r}}$ if for any $\mathrm{f} \in \mathrm{H}^{\mathrm{r}}$, the $n^{\text {th }}$ order approximation $\mathrm{P}_{n} \mathrm{f}$ satisfies

$$
\left\|E_{n} f\right\|_{\infty} \equiv\left\|\left(I-P_{n}\right) f\right\|_{\infty}=O\left(2^{-n s}\right),
$$

as $n$ tends to infinity. It yields best order of approximation $s$ in $H^{r}$ if $s$ is the largest number such that (1.5) holds for all $f \in H^{r}$. If the supremum $s$ of the numbers for which (1.5) holds is not attained we denote the best order of convergence by $s^{-}$. More generally the wavelets $\psi^{\lambda}$ yield pointwise order of approximation (convergence) $\mathrm{s}$ if for any function $\mathrm{f}$ in a sufficiently smooth Sobolev space $H^{r}$ the equality above holds.

Note that by Proposition 2.6 statement (1.5) is equivalent to

$$
\left\|E_{n} f\right\|_{\infty} \leq C_{f} 2^{-n s}\|f\|
$$

for $f \in H^{r}$

In practice (according to the proof of Theorem 1 below) $f$ is sufficiently smooth if $\mathrm{f} \in \mathrm{H}^{\mathrm{s}+\mathrm{d} / 2}$. This is natural since some statements do not make sense for less smooth functions. For example, if the allowed Sobolev exponent were $s+d / 2-\epsilon$ for some $\epsilon>0$, then for small $\mathrm{s}$ functions in $\mathrm{H}^{\mathrm{s}+\mathrm{d} / 2-\epsilon}$ might be discontinuous and the above statements could not hold.

We assume our wavelets satisfy $\psi(\mathrm{x}) \in[\mathrm{RB}]$, the class of radially bounded decreasing $L^{1}$ functions, and/or that our scaling functions are in [RB]. This class of wavelets includes $\mathrm{r}$-regular wavelets (see $[\mathrm{Me}]$ ) for any $\mathrm{r} \geq 0$. The assumptions are needed for appropriate $\mathrm{L}^{\mathrm{p}}$ and a.e. convergence properties of wavelet expansions [KKR1]. 
All the conditions in Theorem 1 below are equivalent, and some (where indicated) require existence of wavelets and/or scaling functions. The wavelet and scaling function assumptions are independent. The conditions focus on the relation of the wavelet's smoothness to convergence rates of wavelet expansions. Note $f(x) \in H_{h}^{s}$ if and only if $\widehat{f}(\xi) \in L^{2}\left(|\xi|^{2 s} d \xi\right)$.

Theorem 1: Given a multiresolution analysis with either $(i)$ a scaling function $\phi \in[\mathrm{RB}]$, (ii) basic wavelets satisfying $\psi^{\lambda}(x) \in[\mathrm{RB}]$ or (iii) a kernel for the basic projection $P$ satisfying $|P(x, y)| \leq K(x-y)$ with $K \in[\mathrm{RB}]$, the following conditions (a to $d^{\prime \prime \prime}$ ) are equivalent for $s>\mathrm{d} / 2$ (existence of a scaling function $\phi$ for the MRA is not assumed in parts (a)-(c') ):

(a) The multiresolution approximation yields pointwise order of approximation $s-d / 2$ in $H^{\mathrm{s}}$, where $d$ denotes dimension.

$\left(a^{\prime}\right)$ The multiresolution approximation yields pointwise order of approximation $r-d / 2$ in $H^{\mathrm{r}}$ for all $r \leq s$ (with $\left.r>d / 2\right)$.

$\left(a^{\prime \prime}\right)$ The multiresolution approximation yields best pointwise order of approximation $s-d / 2$ in $H^{s}$.

$\left(a^{\prime \prime \prime}\right)$ The multiresolution approximation yields best pointwise order of approximation $r-d / 2$ in $H^{\mathrm{r}}$ for all $r \leq \mathrm{s}($ with $r>d / 2)$.

(b) The projection I- $P_{n}: H_{h}^{s} \rightarrow L^{\infty}$ is bounded, where I is the identity.

If there exists a family $\left\{\psi^{\lambda}\right\}$ of basic wavelets corresponding to $\left\{P_{n}\right\}$ with $\psi^{\lambda}(x) \in[\mathrm{RB}]$ (regardless of existence of a scaling function):

(c) For every such family of basic wavelets and each $\lambda, \psi^{\lambda} \in H_{h}^{-s}$, the dual of $H_{h}^{s}$.

$\left(c^{\prime}\right)$ For every such family of basic wavelets and for each $\lambda$,

$$
\int_{|\xi|<\delta}\left|\hat{\psi^{\lambda}}(\xi)\right|^{2}|\xi|^{-2 s} \mathrm{~d} \xi<\infty
$$

for some (or for all) $\delta>0$ (including $\delta=\infty$ ).

$\left(c^{\prime \prime}\right)$ For some such family of basic wavelets, (1.6a) holds.

If there exists a scaling function $\phi$ corresponding to $\left\{P_{n}\right\}$ (with or without a family of wavelets):

(d) For every scaling function $\phi \in[\mathrm{RB}]$ corresponding to $\left\{P_{n}\right\}$

$$
\int_{|\xi|<\delta}\left(1-(2 \pi)^{\mathrm{d} / 2}|\hat{\phi}(\xi)|\right)|\xi|^{-2 \mathrm{~s}} \mathrm{~d} \xi<\infty
$$

for some (or all) $\delta>0$ (including $\delta=\infty$ ).

$\left(d^{\prime}\right)$ For some scaling function $\phi$ corresponding to $\left\{P_{n}\right\}$, (1.6b) holds.

$\left(d^{\prime \prime}\right)$ For every scaling function $\phi \in[\mathrm{RB}]$ corresponding to $\left\{P_{n}\right\}$

$$
\int_{|\xi|<\delta} \sum_{\ell \neq 0}|\hat{\phi}(\xi+2 \pi \ell)|^{2}|\xi|^{-2 s} \mathrm{~d} \xi<\infty
$$

Note that for full generality of the statement of this theorem, the order of proof of the above equivalences includes a direct proof of the implication (b) $\Rightarrow(c)$. Explicit proof of 
this (as opposed to following a different route in the equivalences - see below) is relevant to the case in which we have orthonormal wavelets, but possibly no scaling function. Our direct proof of $(d) \Rightarrow$ (a) will be relevant for the case where there is an MRA, but no orthonormal wavelets which satisfy the $L^{1}$ radial bound conditions required in our proofs; this allows existence of wavelets and scaling functions to be independent.

When a scaling function and wavelet family constructed from this scaling function as in [Me, Gr] (see below) satisfy the same $L^{1}$ radial bounds as the scaling function, Theorem 1 can be more easily proved through the sequence $(d) \Rightarrow(c) \Rightarrow(a) \Leftrightarrow(b)$ and (a) $\Rightarrow$ (d). To be specific, the implication (d) $\Rightarrow$ (c) can be proved more easily if a wavelet basis $\left\{\psi^{\lambda}\right\}_{\lambda}$ constructed from the scaling function (see [Me, Gr, D2]) happens to satisfy our radial bound conditions. Note that for this latter order of proof the radial bound requirement on wavelets is necessary even though the equivalence (a) $\Leftrightarrow$ (d) does not otherwise rely on the existence of a wavelet basis, radially bounded or not. Existence of such wavelets in general (though without guarantees on radial bounds) is proved in [Wo]. If such wavelets satisfy radial bound conditions, we can use that $\psi^{\lambda}(\xi)=m^{\lambda}(\xi) \phi(\xi)$ for some periodic $L^{2}$ function $m^{\lambda}$, and that $\sum_{\lambda}\left|m^{\lambda}(\xi)\right|^{2}=1$, from which we can deduce $(\mathrm{d}) \Rightarrow$ (c) through a simple argument.

We have chosen not to make such assumptions (i.e., the automatic radial boundedness of $\psi^{\lambda}$ following from that of $\phi$ ) in the theorem, and hence our order of proof, which thus must include explicit proofs of $(b) \Rightarrow$ (c) and (d) $\Rightarrow$ (a). We will exclude details of the additional elements of proof for this longer and more general sequence of implications in order to limit the paper's length, and so only sketch the proof of Theorem 2.15 (covering the proof of $(b) \Rightarrow(c)$ ), and sketch the portion of the proof of Theorem 3.7 giving (d) $\Rightarrow$ (a).

We remark $\mathrm{H}^{\mathrm{s}}$ is the "critical" space for order of approximation $\mathrm{s}-d / 2$, in that is the lowest order Sobolev space in which this approximation order can occur.

Condition (b) can refer to any or to all $\mathrm{n}$, since $P_{n}$ are equivalent under scale transformations.

Let $F=\{0,1\}^{\mathrm{d}}$ be the $d$-vectors with entries from the pair $\{0,1\}$. Let

$$
F^{\prime} \equiv F \backslash\{0\},
$$

and recall $m_{0}(\xi)$ is defined in (1.3) (see also Definition 1.1).

Theorem 2: If $m_{0}(\xi)$ is a symbol of a multiresolution analysis corresponding to a sequence of projections as in Theorem 1, the following conditions are equivalent to those in Theorem 1 :

(e) For every symbol $m_{0}(\xi)$ corresponding to $\left\{P_{n}\right\}$,

$$
\int_{|\xi|<\delta}\left(1-\left|\mathrm{m}_{0}(\xi)\right|^{2}\right)|\xi|^{-2 \mathrm{~s}} \mathrm{~d} \xi<\infty
$$

for some (or all) $\delta>0$ (including $\delta=\infty$ ).

$\left(e^{\prime}\right)$ For some symbol $m_{0}(\xi)$ corresponding to $\left\{P_{n}\right\}$, (1.6d) holds.

$\left(e^{\prime \prime}\right)$ Every (or some) symbol $m_{0}(\xi)$ corresponding to $\left\{P_{n}\right\}$ satisfies 


$$
\int_{|\xi-\pi \epsilon|<\delta}\left|m_{0}(\xi)\right|^{2}|\xi-\pi \epsilon|^{-2 s} d \xi<\infty
$$

for some (or all) $\delta>0$ (including $\delta=\infty$ ) and for every $\epsilon \in F^{\prime}$.

Again the above hold for multiresolution expansions, scaling expansions, and wavelet expansions if defined.

\section{Remarks:}

1. The above conditions are related to Strang-Fix conditions [SF], which relate $\mathrm{H}^{\mathrm{s}}$ and $\mathrm{L}^{\infty}$ orders of convergence of spline expansions to moment and other conditions, and our theorem is effectively an extension of the so-called Condition A in [Str]. A significant difference is that our results yield fractional orders of convergence.

Fractional convergence orders are in fact possible for multiresolution expansions. Indeed, in Theorem 1 it is shown order of convergence of a wavelet expansion is determined by the asymptotics of the wavelet's (scaling function's) Fourier transform near the origin. To show any convergence order is possible, it suffices to construct scaling functions with arbitrary asymptotics near the origin (not just those corresponding to integral orders $s-d / 2$ of convergence).

2. Alternatively, condition (c) above is equivalent to $|\Delta|^{-(1 / 2)(\mathrm{s})} \psi^{\lambda} \in \mathrm{L}^{2}\left(\mathbb{R}^{\mathrm{d}}\right)$, with $|\Delta|$ defined by operator calculus. This is a singular integral condition on $\psi$ itself (e.g., [Ste]). Thus

$$
\psi^{\lambda}=|\Delta|^{\mathrm{s} / 2} \eta^{\lambda},
$$

for some $\eta^{\lambda} \in \mathrm{L}^{2}\left(\mathbb{R}^{\mathrm{d}}\right)$.

For $d=1$ and $s=k$ an even integer, the standard assumption that for some $\epsilon>0$

$$
|\psi(\mathrm{x})| \leq \mathrm{C}(1+|\mathrm{x}|)^{-\mathrm{k}-1-\epsilon},
$$

implies $\left|\eta^{(\ell)}(\mathrm{x})\right| \leq \mathrm{C}(1+|\mathrm{x}|)^{-\ell-1-\epsilon}$ for integers $\ell \leq \mathrm{k}$. So it can be shown ([KR2]) that (c) in Theorem 1 is equivalent to the moment condition

$$
\int \mathrm{x}^{\ell} \psi(\mathrm{x}) \mathrm{dx}=0 \quad \text { for } \quad 0 \leq \ell \leq \mathrm{k}-1 .
$$

The latter is equivalent to other versions of the Strang-Fix conditions [SF].

3. Condition (1.6b) is related to $r$-regularity. Using Proposition 2.7 in [Me] we can conclude from our theorem pointwise convergence of order $r+1-\epsilon$ for r-regular expansions in one dimension, for all $\epsilon>0$.

4. The $s$ range in the theorem cannot be extended. Indeed if $s \leq d / 2$ then $I-P: H^{s} \rightarrow L^{\infty}$ cannot be bounded, as shown in a remark below. On the other hand for $s<d / 2$ conditions $\left(c^{\prime}\right)$ and $\left(d^{\prime}\right)$ of the above theorem always hold. Indeed this is clear by 
the boundedness of $\psi$ and $\phi$. Since the statements of the above theorems certainly make sense for all $s>0$ (but are false for $s<d / 2$ ), the proofs of the conditions on $\psi^{\lambda}$ and $\phi$ must be delicate enough that they fail when $s<d / 2$. The points of failure are indicated in the proofs below.

5. The scaling factors of 2 in the spaces $V_{j}$ is not crucial; the arguments hold for other scalings, as long as a multiresolution analysis of the full function space results (see $[\mathrm{Au}]$ ).

Our strategy when wavelets are assumed to exist is to form of the kernel of $P_{n}$, given by

$$
\mathrm{P}_{\mathrm{n}}(\mathrm{x}, \mathrm{y})=\sum_{\mathrm{j}<\mathrm{n} ; \mathrm{k} ; \lambda} \psi_{\mathrm{jk}}^{\lambda}(\mathrm{x}) \overline{\psi_{\mathrm{jk}}^{\lambda}}(\mathrm{y})
$$

A similar expression in terms of the scaling function $\phi$ will also be useful.

Finally, the present results are best possible $L^{\infty}$ results in the scales of Sobolev spaces. Note we assume both functions to be expanded and wavelets and scaling functions are in $L^{2}$. This corollary follows immediately from Theorems 1 and 2:

Corollary 3: (a) Given the Sobolev space $H^{s}$ as the space of functions $f$ to be expanded, the homogeneous space $H_{h}^{-s}$ is the maximal space of possible wavelet functions $\psi^{\lambda}$ (if they exist) for which Theorems 1 and 2 hold.

(b) Given the Sobolev space $H^{s}$ as the space of functions $f$ to be expanded, $H_{h}^{-s}$ is the maximal space of the function $1-(2 \pi)^{d / 2} \widehat{\phi}$ (if $\phi$ exists) for which Theorems 1 and 2 hold. (c) Given $H_{h}^{-s}$ as the maximal space (i.e., smallest s) to which the $\psi^{\lambda}\left(\right.$ or $\left.1-(2 \pi)^{d / 2} \phi\right)$ belongs, the maximal Sobolev space of expanded functions $f$ for which Theorems 1 and 2 hold (i.e., for which we have order or best order of approximation $s-d / 2$ ) is $H^{s}$.

For completeness in section 5 we show convergence rates of wavelet expansions, though they must be exponential in almost all spaces, can be arbitrarily slow within this constraint.

\section{Rates of convergence and wavelets}

We restate needed results from [KKR1]; $\phi$ and $\psi$ denote the scaling function and basic wavelet of a multiresolution expansion. Statements are in $\mathbb{R}^{\mathrm{d}}$ unless indicated otherwise.

Lemma 2.1 [KKR1]: (i) If the scaling function $\phi \in[\mathrm{RB}]$, then the kernel $P(\mathrm{x}, \mathrm{y}) \equiv$ $\sum_{\mathrm{k} \in \mathbb{Z}^{\mathrm{d}}} \phi(\mathrm{x}-\mathrm{k}) \bar{\phi}(\mathrm{y}-\mathrm{k})$ satisfies

$$
|\mathrm{P}(\mathrm{x}, \mathrm{y})| \leq K_{0}(\mathrm{x}-\mathrm{y})
$$

where $K_{0} \in[\mathrm{RB}]$, i.e., is a bounded radial decreasing $L^{1}$ function. Convergence of this sum is uniform on $\mathbb{R}^{2 d}$ and the sum is the $L^{2}$ kernel of $P_{0}$. 
(ii) If $\psi^{\lambda}(\mathrm{x}) \in[\mathrm{RB}]$, then $Q(x, y) \equiv \sum_{k \in \mathbb{Z}^{d} ; \lambda} \psi^{\lambda}(x-k) \overline{\psi^{\lambda}}(y-k)$ converges uniformly and absolutely on $\mathbb{R}^{2 d}$, and is bounded. This is the kernel of the orthogonal projection $Q_{0}$ onto $W_{0}$.

A Lebesgue point $x$ of a function $f$ is a generalized continuity point near which $\mathrm{f}$ does not deviate too much from the value $\mathrm{f}(\mathrm{x})$ :

Definition 2.2: The point $\mathrm{x}$ is a Lebesgue point of the measurable function $\mathrm{f}(\mathrm{x})$ on $\mathbb{R}^{\mathrm{d}}$ if $\mathrm{f}$ is integrable in some neighborhood of $\mathrm{x}$ and

$$
\lim _{\delta \rightarrow 0} \frac{1}{V\left(B_{\delta}\right)} \int_{B_{\delta}}|f(x)-f(x+y)| d y=0,
$$

where $\mathrm{B}_{\delta}$ denotes the ball of radius $\delta$ about the origin, and $\mathrm{V}$ is volume.

Continuity points are also Lebesgue points, though the Lebesgue set can be much larger than the continuity set. Lebesgue points have full Lebesgue measure on $\mathbb{R}^{d}$.

Theorem 2.3 [KKR1]: (i) Assume only that the scaling function $\phi$ of a given multiresolution analysis is in $[\mathrm{RB}]$, i.e. that it is bounded by an $L^{1}$ radial decreasing function. Then for an $f \in L^{p}\left(\mathbb{R}^{d}\right) \quad(1 \leq \mathrm{p} \leq \infty)$, its multiresolution approximation converges to $f$ pointwise almost everywhere.

(ii) If $\phi, \psi^{\lambda} \in[\mathrm{RB}]$ for all $\lambda$, then also the scaling (1.4b) (if $1 \leq p \leq \infty$ ) and wavelet (1.4a) (if $1 \leq p<\infty$ ) expansions of any $f \in \mathrm{L}^{\mathrm{p}}\left(\mathbb{R}^{\mathrm{d}}\right)$ converge to $f$ pointwise almost everywhere. If further $\phi$ and $\psi^{\lambda}$ are (partially) continuous, then both of these expansions additionally converge to fon its entire Lebesgue set.

(iii) If we assume only $\psi^{\lambda}(x) \ln (2+|\mathrm{x}|) \in[\mathrm{RB}]$ for all $\lambda$, then the wavelet (for $\left.1 \leq \mathrm{p}<\infty\right)$ and multiresolution (for $1 \leq \mathrm{p} \leq \infty$ ) approximations of any $f \in \mathrm{L}^{\mathrm{p}}\left(\mathbb{R}^{\mathrm{d}}\right.$ ) converge to $f$ pointwise almost everywhere; if further the $\psi^{\lambda}$ are (partially) continuous, then the wavelet and multiresolution expansions converge to fon its Lebesgue set.

(iv) The last two statements hold for orders of summation where, at any stage, the range of the values of $j$ for which the sum over $k$ and $\lambda$ is partially complete always remains bounded.

Definition 2.4: Two functions $\alpha(\cdot)$ and $\beta(\cdot)$ are equivalent, $\alpha(\cdot) \sim \beta(\cdot)$, if there exist positive constants $\mathrm{c}_{1}$ and $\mathrm{c}_{2}$ such that for every $f$ in their domain,

$$
\mathrm{c}_{1} \alpha(f) \leq \beta(f) \leq \mathrm{c}_{2} \alpha(f)
$$

The $\mathrm{L}^{2}$-Sobolev norm is equivalent to a more convenient one. The homogeneous norm (Def. 1.2) has better scaling properties, and a relationship is:

$$
\|\mathrm{f}\|_{\mathrm{s}} \sim\|\mathrm{f}\|_{\mathrm{h}, \mathrm{s}}+\|\mathrm{f}\|_{0} \equiv\|f\|_{\widetilde{H}^{s}}
$$

for $s>0$. 
Definition 2.5: Let $\widetilde{H^{s}}$ be the space $H^{s}$ endowed with the equivalent norm $\|\cdot\|_{\widetilde{H}^{s}}$ above.

If $\mathcal{F}$ is Fourier transform, then $\mathcal{F}(\mathrm{f}(\mathrm{ax}))=\mathrm{a}^{-\mathrm{d}} \hat{\mathrm{f}}(\xi / \mathrm{a})$. Letting

$$
f_{n}(x)=2^{n d / 2} f\left(2^{n} x\right),
$$

we have

$$
\begin{gathered}
\left\|\mathrm{f}_{\mathrm{n}}\right\|_{\mathrm{h}, \mathrm{s}}^{2}=\int|\xi|^{2 \mathrm{~s}}\left(2^{\mathrm{nd} / 2} 2^{-\mathrm{nd}}\left|\hat{\mathrm{f}}\left(2^{-\mathrm{n}} \xi\right)\right|\right)^{2} \mathrm{~d} \xi \\
=2^{2 \mathrm{~ns}}\|\mathrm{f}\|_{\mathrm{h}, \mathrm{s}}^{2} .
\end{gathered}
$$

A key to our discussion is that convergence rates are closely related to the behavior of Fourier transforms of wavelets and scaling functions (and more generally any dilated and translated expansion functions) near the origin. This behavior translates to that of the kernels $P_{n}$ and $Q_{n}$ of the projections onto $V_{n}$ and $W_{n}$. We inverse Fourier transform these kernels and define

$$
\tilde{\mathrm{P}_{\mathrm{n}}}(\mathrm{x}, \xi)=\mathcal{F}_{\mathrm{y}}^{-1} \mathrm{P}_{n}(\mathrm{x}, \mathrm{y}) ; \quad \widetilde{\mathrm{Q}_{\mathrm{n}}}(\mathrm{x}, \xi)=\mathcal{F}_{\mathrm{y}}^{-1} \mathrm{Q}_{n}(\mathrm{x}, \mathrm{y}),
$$

where the subscript indicates the inverse Fourier transform is in y. It is easy to verify the transforms converge everywhere and are continuous in $\xi$, since under the assumption $\phi \in[\mathrm{RB}]$ it follows $P_{n}(x, y)$ and $Q_{n}(x, y) \in \mathrm{L}_{\mathrm{y}}^{1}$, uniformly in x. The same conclusions hold for $Q_{n}(x, y)$ if $\psi \in[\mathrm{RB}]$ (see [KKR1]).

We effectively study the distance of the kernel $P_{n}(x, y)$ to the delta distribution $\delta(x-y)$ in $H^{-s}(s>d / 2)$ to prove our results (this viewpoint is taken in [Wa]). Consider the error

$$
\left\|E_{\mathrm{n}}\right\|=\left\|\delta(x-y)-\mathrm{P}_{\mathrm{n}}(\mathrm{x}, \mathrm{y})\right\|_{\mathrm{H}^{\mathrm{s}} \rightarrow \mathrm{L}^{\infty}}
$$

where $\delta(\mathrm{x}-\mathrm{y})$ is viewed as a convolution "kernel" applicable to functions in sufficiently smooth Sobolev spaces. Strictly speaking the latter norm is that of the operator $I-P_{\mathrm{n}}$, since $\delta$ does not exist as a function.

The error $E_{n}$ is bounded in $L^{2}$, but has no $L^{2}$ kernel in $x$ and $y$ since that of $\mathrm{I}$ is a delta distribution. In Fourier space the kernel is well defined:

$$
\mathrm{E}_{\mathrm{n}} \mathrm{f}=\tilde{E}_{\mathrm{n}} \mathcal{F f},
$$

where $\widetilde{E_{\mathrm{n}}}$ has a kernel $\widetilde{E}_{n}(x, \xi)=(2 \pi)^{-\mathrm{d} / 2} \mathrm{e}^{\mathrm{ix} \xi}-\tilde{\mathrm{P}_{\mathrm{n}}}(\mathrm{x}, \xi)$, with

$$
\widetilde{P}_{n}(x, \xi)=\widehat{P}_{n}(x,-\xi)=(2 \pi)^{-d / 2} \int P_{n}(x, y) e^{i \xi y} d \xi
$$

Now we equate convergence orders and statements about operator norms:

Proposition 2.6: Given a Banach space $A$ and a normed linear space $B$, and a sequence of operators $T_{n}: A \rightarrow B$, the sequence has order of approximation $\beta(n)$, i.e., for all $f \in A$, $\left\|\left(I-T_{n}\right) f\right\|_{B} \leq C_{f} \beta(n)$ if and only if the operator norm $\left\|I-T_{n}\right\| \leq C^{\prime} \beta(n)$. 
Proof: Consider the operators $A_{n} \equiv\left(I-T_{n}\right) / \beta(n)$. The second condition above states that $A_{n}$ are uniformly bounded. By the uniform boundedness principle, this occurs if and only if $\left\|\left(I-T_{n}\right) f / \beta(n)\right\|_{B}$ is bounded in $n$ for every $f \in A$, completing the proof.

Define the scaling operator $S f(x)=f(2 x) . \quad$ Then $\quad V_{n}=S V_{n-1}$, and $P_{n}=S P_{n-1} S^{-1}=S^{n} P_{0} S^{-n}$. We have also

$$
P_{n}(x, y)=2^{n d} P_{0}\left(2^{n} x, 2^{n} y\right)
$$

Let $s>0$ and let $\mathrm{E}_{\mathrm{n}}=\mathrm{I}-\mathrm{P}_{\mathrm{n}}$ denote the error operator for $V_{n}$; let $E \equiv E_{0}$.

Lemma 2.7: The sequence $2^{-n(d / 2-s)}\left\|E_{n}\right\|_{\widetilde{H}^{s} \rightarrow L^{\infty}}$ is nondecreasing, with limit $\|E\|_{H_{h}^{s} \rightarrow L^{\infty}}$ (which could be infinite).

Proof: For $f \in H^{s}$, define $f_{n}(x)=2^{n d / 2} f\left(2^{n} x\right)$. Note $\left\|f_{n}\right\|_{0}=\|f\|_{0}$, as well as $\left\|f_{n}\right\|_{h, s}=2^{n s}\|f\|_{h, s}$, and $\left(E_{n} f_{n}\right)(x)=2^{\text {nd } / 2}(E f)\left(2^{n} x\right)$. Thus

$$
\left\|\mathrm{E}_{n} f_{n}\right\|_{L^{\infty}}=2^{n d / 2}\|E f\|_{L^{\infty}} .
$$

Let $A \equiv\|E\|_{H_{h}^{s} \rightarrow L^{\infty}}$ and

$$
a_{n} \equiv \frac{\left\|E_{n}\right\|_{\widetilde{H}^{s} \rightarrow L^{\infty}}}{2^{n(d / 2-s)}}=\sup _{f_{n} \in H^{s}} \frac{\left\|E_{n} f_{n}\right\|_{L^{\infty}}}{2^{n(d / 2-s)}\left(\left\|f_{n}\right\|_{H_{h}^{s}}+\left\|f_{n}\right\|_{0}\right)}=\sup _{f \in H^{s}} \frac{\|E f\|_{L^{\infty}}}{\left(\|f\|_{H_{h}^{s}}+2^{-n s}\|f\|_{0}\right)} .
$$

This last expression shows that $\left\{a_{n}\right\}_{n \in \mathbb{Z}}$ is a nondecreasing sequence bounded above by $A$ (since the sets $H^{s}$ and $H_{h}^{s}$ are defined to be the same). Hence $a_{n}$ converges (possibly to $+\infty)$ and $\lim _{n \rightarrow \infty} a_{n} \leq A$.

Conversely, let $f \in H_{h}^{s}$ (so $f \in L^{2}$ by definition). Then by definition of $a_{n}$,

$$
\begin{aligned}
\|E f\|_{L^{\infty}}=2^{-n d / 2}\left\|E_{n} f_{n}\right\|_{L^{\infty}} \leq a_{n} 2^{-n s}\left\|f_{n}\right\|_{\widetilde{H}^{s}} & =a_{n} 2^{-n s}\left(\left\|f_{n}\right\|_{H_{h}^{s}}+\left\|f_{n}\right\|_{0}\right) \\
& =a_{n}\left(\|f\|_{H_{h}^{s}}+2^{-n s}\|f\|_{0}\right),
\end{aligned}
$$

for each $n$. Let $n \rightarrow \infty$ to conclude that $\|E f\|_{L^{\infty}} \leq\|f\|_{H_{h}^{s}} \lim _{n \rightarrow \infty} a_{n}$. Hence $A \leq \lim _{n \rightarrow \infty} a_{n}$.

Recall an operator $T$ is in [RB] if it has a kernel $|T(x, y)| \leq K(x-y)$ with $K(\cdot)$ $\in[\mathrm{RB}]$. Since $\widetilde{H}^{s}$ and $H^{s}$ norms are equivalent we have by the Lemma:

Proposition 2.8: Assume $P=P_{0} \in[\mathrm{RB}]$ and let $s>0$. Then

(a) The error $E_{n}=I-P_{n}$ satisfies the scaling identity

$$
\left\|E_{n}\right\|_{H^{s} \rightarrow L^{\infty}} \sim 2^{-n(s-d / 2)}\|E\|_{H_{h}^{s} \rightarrow L^{\infty}},
$$

with the equivalence uniform over $n$, if the right side is finite.

(b) If the right side of (2.3) is infinite then 


$$
\frac{\left\|E_{n}\right\|_{H^{s} \rightarrow L^{\infty}}}{2^{-n(s-d / 2)}} \underset{n \rightarrow \infty}{\rightarrow \infty}
$$

Thus if (2.3) is finite for some $n$,

$$
\mathrm{C}_{1} 2^{\mathrm{n}(\mathrm{d} / 2-\mathrm{s})}\|\mathrm{E}\|_{\mathrm{H}_{\mathrm{h}}^{\mathrm{s}} \rightarrow \mathrm{L}^{\infty}}<\left\|\mathrm{E}_{\mathrm{n}}\right\|_{\mathrm{H}^{\mathrm{s}} \rightarrow \mathrm{L}^{\infty}}<\mathrm{C}_{2} 2^{\mathrm{n}(\mathrm{d} / 2-\mathrm{s})}\|\mathrm{E}\|_{\mathrm{H}_{\mathrm{h}}^{\mathrm{s}} \rightarrow \mathrm{L}^{\infty}} .
$$

Taking (a) and noting (b) of Proposition 2.8 when $\|\mathrm{E}\| \mathrm{H}_{\mathrm{h}}^{\mathrm{s}} \rightarrow \mathrm{L}^{\infty}=\infty$, we obtain:

Theorem 2.9 $((\mathrm{a}) \Leftrightarrow(\mathrm{b})$ in Theorem 1): Consider a multiresolution analysis with reproducing projections $P_{\mathrm{n}}$ onto $V_{\mathrm{n}}$ which are in $[\mathrm{RB}]$, and let $s>0$. A necessary and sufficient condition that this multiresolution analysis yield pointwise approximations of order $s-\mathrm{d} / 2$ in $H^{\mathrm{s}}$ is that $I-P: \mathrm{H}_{\mathrm{h}}^{\mathrm{s}} \rightarrow \mathrm{L}^{\infty}$ be bounded, with $P$ the projection onto $V_{0}$, and $I$ the identity.

Remarks: 1. Since $\mathrm{P}$ is an operator on $\mathrm{L}^{2}$, Theorem 2.9 technically states $I-P$ maps $\mathrm{H}_{\mathrm{h}}^{\mathrm{s}+\mathrm{d} / 2} \cap \mathrm{L}^{2}$ into $\mathrm{L}^{\infty}$, though since $\mathrm{L}^{2} \cap \mathrm{H}_{\mathrm{h}}^{\mathrm{s}}$ is dense in $\mathrm{H}_{\mathrm{h}}^{\mathrm{s}}$, the statements are equivalent.

2. Since $\mathrm{s} \leq d / 2$ is allowed in Theorem 2.9 , this also formally describes situations in which pointwise approximations are guaranteed to diverge at the rate $\mathrm{O}\left(2^{\mathrm{n}(\mathrm{d} / 2-\mathrm{s})}\right)$ for some functions f. This may apply to some multiresolution expansions, but for wavelet expansions $\mathrm{s} \leq d / 2$ is vacuous, since in this case $I-P: \mathrm{H}_{\mathrm{h}}^{\mathrm{s} d / 2} \rightarrow \mathrm{L}^{\infty}$ is never bounded. Indeed we assume the basic wavelet $\psi$ is bounded, so $\mathrm{Pf} \in \mathrm{L}^{\infty}$ for $\mathrm{f} \in \mathrm{L}^{2}$. On the other hand there exist unbounded functions in $\mathrm{H}^{\mathrm{s}}$ and hence $\mathrm{H}_{\mathrm{h}}^{s}$ for $\mathrm{s} \leq d / 2$. Hence for $\mathrm{s} \leq$ $d / 2$ it is impossible for $(P-I) f$ to be bounded for all $\mathrm{f} \in \mathrm{H}_{\mathrm{h}}^{\mathrm{s}}$ and the hypothesis of the theorem is never satisfied in this case.

We can in fact prove a more general theorem. For this we need:

Definition 2.10: $\quad$ Let $A$ be a normed linear space (NLS) of functions on a vector space $X$ with norm $\|\cdot\|_{\mathrm{a}}$. Define the associated homogeneous norm $\|\cdot\|_{\mathrm{a}, \mathrm{h}}$ by

$$
\|\mathrm{f}\|_{\mathrm{a}, \mathrm{h}}=\lim _{\mathrm{c} \rightarrow \infty}\|\mathrm{f}(\mathrm{cx})\|_{\mathrm{a}} / \alpha(\mathrm{c})
$$

where $\alpha(\mathrm{c})$ is a positive function (if it exists) for which the limit (2.4) exists for all $\mathrm{f} \in \mathrm{A}$, and is nontrivial for $f \neq 0$. An NLS $A$ for which there exists $\alpha(c)$ such that $\|f(c x)\|_{a}=\alpha(\mathrm{c})\|f(x)\|$ for all $c>0$ is a homogeneous space.

The norm (2.4) is uniquely defined up to a constant multiple for all $\alpha$, if any $\alpha$ yielding a homogeneous norm exists. Indeed if $\alpha_{1}$ and $\alpha_{2}$ are two such functions the ratio of the resulting norms will be $\lim _{\mathrm{c} \rightarrow \infty} \alpha_{1}(\mathrm{c}) / \alpha_{2}$ (c). If $\alpha(c)=c^{k}$, then $k$ is the scaling factor of $A$.

Note $\alpha(c)$ need not be a pure power of $c$. If $\|f(x)\| \equiv \int|f(x)|(|\ln x|+1) d x$,

$$
\|f(c x)\|=c^{-1} \int|f(x)|(|\ln (x / c)|+1) d x \sim c^{-1} \ln c \int|f(x)| d x \quad(c \rightarrow \infty),
$$


so $\alpha(c)=c^{-1} \ln c$, and $\|f\|_{a, h}=\|f\|_{1}$.

We remark that associated homogeneous norms exist for all $\mathrm{L}^{\mathrm{p}}-$ Sobolev spaces $L_{s}^{p}$, $1 \leq \mathrm{p} \leq \infty$, and that for $L_{s}^{p}$ we have $\alpha(\mathrm{c})=\mathrm{c}^{s-\mathrm{d} / \mathrm{p}}$.

Theorem 2.11: Let $A$ and $\mathrm{B}$ be any homogeneous Banach spaces of functions on $\mathbb{R}^{d}$, with scaling factors $k_{A}$ and $k_{B}$. Then a necessary and sufficient condition for a multiresolution analysis $\left\{P_{\mathrm{n}}\right\}$ to yield approximations of order $\mu=k_{B} k_{A}^{-1}$ from the space A to the space $B$ is that $I-P: \mathrm{A} \rightarrow \mathrm{B}$ be bounded.

Proof: Letting $E_{n}=I-P_{n}$

$$
\begin{gathered}
\left\|E_{n}\right\|_{A \rightarrow B}=\left\|S^{n} E S^{-n}\right\|_{A \rightarrow B}=\sup _{f \in A}\left\|S^{n} E S^{-n} f\right\|_{B} /\|f\|_{A} \\
=\sup _{f \in A}\left\|S^{n} E f\right\|_{B} /\left\|S^{n} f\right\|_{A}=k_{B}^{n} k_{A}^{-n} \sup _{f \in A}\|E f\|_{B} /\|f\|_{A} .
\end{gathered}
$$

Thus if $E=I-P$ is bounded we get approximations of the desired order. Conversely if $E$ is unbounded then so is $E_{n}$, and so by Proposition 2.6 we fail to have any order of convergence.

Thus order of approximation between homogeneous spaces is entirely determined by the scaling factors (if they exist) of the two spaces A and B.

We now consider what properties of the basic wavelet $\psi$ imply order $s$ convergence using Theorem 2.9. The correct condition will be that $\psi$ be in the dual space $H_{h}^{s *}=H_{h}^{-s}$. Note that

$$
(2 \pi)^{\mathrm{d} / 2} \mathcal{F}\left(\psi_{\mathrm{jk}}^{\lambda}(\mathrm{x})\right)=2^{-\mathrm{jd} / 2} \mathrm{e}^{-\mathrm{i} 2^{-\mathrm{j}} \xi \mathrm{k}}(2 \pi)^{\mathrm{d} / 2} \hat{\psi}^{\lambda}\left(2^{-\mathrm{j}} \xi\right) .
$$

Theorem $2.12((\mathrm{c}) \Rightarrow(\mathrm{a}))$ : Let $s>d / 2$ and assume $\psi^{\lambda}(x) \in[\mathrm{RB}]$ and $\psi^{\lambda} \in H_{h}^{-s}$ for all $\lambda$. Then the MRA yields pointwise order of approximation $s-d / 2$ in $H^{s}$.

Proof: Suppose $\psi^{\lambda} \in H^{-s}$ for each $\lambda$, and $f \in H^{s}$. Then

$$
\left|\left\langle f, \psi_{j, k}^{\lambda}\right\rangle\right| \leq\|f\|_{H_{h}^{s}}\left\|\psi_{j, k}^{\lambda}\right\|_{H_{h}^{-s}}=2^{-j s}\|f\|_{H_{h}^{s}}\left\|\psi^{\lambda}\right\|_{H_{h}^{-s}},
$$

by the formula (2.5) and a simple dilation argument. Since $\psi^{\lambda} \in[\mathrm{RB}]$,

$$
\sup _{t \in \mathbb{R}^{d}} \sum_{k \in \mathbb{Z}^{d}}\left|\psi^{\lambda}(t-k)\right| \equiv A_{\lambda}<\infty .
$$

Hence for each $\lambda$, each $j \in \mathbb{Z}$, and each $x \in \mathbb{R}^{d}$,

$$
\begin{aligned}
\left|\sum_{k \in \mathbb{Z}}\left\langle f, \psi_{j, k}^{\lambda}\right\rangle \psi_{j, k}^{\lambda}(x)\right| & \leq 2^{-j s}\|f\|_{H_{h}^{s}}\left\|\psi^{\lambda}\right\|_{H_{h}^{-s}} \sum_{k \in \mathbb{Z}^{d}} 2^{j d / 2}\left|\psi^{\lambda}\left(2^{j} x-k\right)\right| \\
& \leq 2^{-j(s-d / 2)} A_{\lambda}\|f\|_{H_{h}^{s}}\left\|\psi^{\lambda}\right\|_{H_{h}^{-s}}
\end{aligned}
$$

Therefore 


$$
\begin{aligned}
\left\|\left(I-P_{n}\right) f\right\|_{\infty} \leq \sum_{\lambda} \sum_{j=n+1}^{\infty}\left\|\sum_{k \in \mathbb{Z}}\left\langle f, \psi_{j, k}^{\lambda}\right\rangle \psi_{j, k}^{\lambda}\right\|_{\infty} & \leq \sum_{\lambda} \sum_{j=n+1}^{\infty} 2^{-j(s-d / 2)} A_{\lambda}\|f\|_{H_{h}^{s}}\left\|\psi^{\lambda}\right\|_{H_{h}^{-s}} \\
& \leq c 2^{-n(s-d / 2)}\|f\|_{H_{h}^{-s}} \sum_{\lambda} A_{\lambda}\left\|\psi^{\lambda}\right\|_{H_{h}^{-s}},
\end{aligned}
$$

as desired.

Corollary $2.13((\mathrm{c}) \Rightarrow(\mathrm{b}))$ : The projection $I-P_{n}: H_{h}^{s} \rightarrow L^{\infty}$ is bounded if $\psi \in[\mathrm{RB}]$ and $\psi \in H_{h}^{-s}$ for each $\lambda$.

Proof: Theorems 2.9 and 2.12.

We now prove (b) implies (c) via:

Lemma 2.14: Let $g_{\mathrm{i}}(\mathrm{n})$ be complex-valued functions of an integer argument $n$ for each $1 \leq \mathrm{i} \leq \ell$, with $\limsup _{\mathrm{n} \rightarrow \infty}\left|\mathrm{g}_{\mathrm{i}}(\mathrm{n})\right|=\infty$ for at least one $i$. Then there exists a vector $v$ such that if we define $\boldsymbol{g}(n)=\left(g_{1}(n), \ldots, g_{\ell}(n)\right)$, then for any vector $\boldsymbol{c}$,

$$
\limsup _{n \rightarrow \infty}|\mathbf{c} \cdot \mathbf{g}(\mathrm{n})|<\infty
$$

only if $\boldsymbol{c} \cdot \boldsymbol{v}=0$.

Proof: Let $\mathbf{h}(\mathrm{n})=\mathbf{g}(\mathrm{n}) /|\mathbf{g}(\mathrm{n})|$, and let $\mathbf{v}$ be a limit point of the sequence $\mathbf{h}(\mathrm{n})$. Then if $\mathbf{c} \cdot \mathbf{v} \neq 0$, we would have for a subsequence $n_{k}$ such that $\mathbf{h}\left(n_{k}\right) \underset{k \rightarrow \infty}{\rightarrow} \mathbf{v},\left|c \cdot \mathbf{h}\left(n_{k}\right)\right|>\epsilon$ for some positive $\epsilon$. Thus we would have

$$
\limsup _{n \rightarrow \infty}|\mathbf{c} \cdot \mathbf{g}(\mathrm{n})|=\limsup _{\mathrm{n} \rightarrow \infty}|\mathbf{c} \cdot \mathbf{h}(\mathrm{n})||\mathbf{g}(\mathrm{n})| \geq \limsup _{\mathrm{n} \rightarrow \infty} \epsilon|\mathbf{g}(\mathrm{n})|=\infty
$$

yielding the desired result.

In the next theorem note $\psi(\mathrm{x}) \in \mathrm{H}_{\mathrm{h}}^{\mathrm{s}^{*}}$ is a condition on $\psi$ as a linear functional. This is equivalent to bounds on the Fourier transform of $\psi$, which dictate the proof's approach. Recall $\mathrm{P}=\mathrm{P}_{0}$ is the $L^{2}$ orthogonal projection onto $\mathrm{V}_{0}$. Announcements of this result have appeared in [KKR1, KR2]; here we include the complete technical details.

The proof of Theorem 2.15 below is sketched (see remark at end of Section 1).

Theorem $2.15\left((\mathrm{~b}) \Rightarrow\right.$ (c) in Theorem 1): Let $\mathrm{s}>\mathrm{d} / 2$ and assume $\psi^{\lambda} \in[\mathrm{RB}]$ for all $\lambda$. Then if $I-P: H_{h}^{s} \rightarrow L^{\infty}$ is bounded, $\psi(\mathrm{x}) \in H_{h}^{s^{*}}$, the dual space of $H_{\mathrm{h}}^{\mathrm{s}}$.

Proof: Assume $\psi^{\lambda_{1}} \notin \mathrm{H}_{\mathrm{h}}^{\mathrm{s}^{*}}=\mathrm{H}_{\mathrm{h}}^{-\mathrm{s}}$ for some $\lambda_{1} \in \Lambda$. Assume $\lambda$ takes values from 1 to $\ell$ and without loss assume $\lambda_{1}=1$. Then there is a sequence $\mathrm{f}_{n}$, with $\widehat{f_{n}}(\xi)$ in $C_{0}^{\infty}$ and $\left\|f_{n}\right\|_{h, \mathrm{~s}}=1$ such that $\int \hat{\psi^{1}}(\xi) \hat{f_{\mathrm{n}}}(\xi) \mathrm{d} \xi \underset{\mathrm{n} \rightarrow \infty}{\rightarrow}+\infty$. The sequence $\hat{\mathrm{f}_{\mathrm{n}}}$ can be chosen so the integrand above is positive for all $\mathrm{n}$. Let $\hat{\mathrm{Q}_{0}}(\mathrm{x}, \xi)$ be the Fourier transform in $y$ of $\mathrm{Q}_{0}(\mathrm{x}, \mathrm{y})$ 
$\equiv \mathrm{Q}(\mathrm{x}, \mathrm{y})$, which exists as a function by the bounds in Lemma 2.1. Using (2.5) we can show (all $\mathrm{L}^{\infty}$ norms are in $\mathrm{x}$ )

$$
\left\|\int \mathrm{Q}_{0}(\mathrm{x}, \mathrm{y}) \mathrm{f}_{\mathrm{n}}(\mathrm{y}) \mathrm{dy}\right\|_{\infty}=\left\|\int \hat{f}_{n}(-\xi) \sum_{\lambda} \overline{\hat{\psi}}^{\lambda}(-\xi) Z^{\lambda}(x, \xi) d \xi\right\|_{\infty}
$$

where

$$
Z^{\lambda}(\mathrm{x}, \xi) \equiv \sum_{\mathrm{k}} \mathrm{e}^{-\mathrm{i} \xi \mathrm{k}} \psi^{\lambda}(x-k)
$$

is the Zak transform of $\psi^{\lambda}$.

We now show the $\mathrm{L}^{\infty}$ norm in (2.6) becomes infinite as $\mathrm{n} \rightarrow \infty$. Note we have assumed that $\psi^{\lambda} \in[\mathrm{RB}] \subset \mathrm{L}^{2}$ (recall functions in $[\mathrm{RB}]$ are bounded), but that

$$
\int \overline{\hat{\psi}^{1}(\xi)} \hat{\mathrm{f}}_{\mathrm{n}}(\xi) \mathrm{d} \xi \underset{\mathrm{n} \rightarrow \infty}{\rightarrow} \infty
$$

It is not difficult to see we can choose $\hat{f_{n}}(\xi)$ to be a sequence of $C_{0}^{\infty}$ functions whose support does not contain the origin (though the origin may be in the limit of the supports of $\hat{f_{n}}$ ). Assume without loss there is a subsequence $\left\{\mathrm{n}_{\mathrm{k}}\right\}$ such that

$$
\int \hat{\psi}^{1}(\xi) \hat{f_{n_{k}}}(\xi) d \xi \geq \int\left|\hat{\psi^{\lambda}}(\xi) \hat{f_{n_{k}}}(\xi)\right| d \xi
$$

for all $\lambda$; otherwise we could change indexing of the $\lambda$ 's so this is the case, and then adjust the phase of the $\mathrm{f}_{\mathrm{n}_{\mathrm{k}}}$ so that $\bar{\psi}^{1}(\xi) \hat{\mathrm{f}}_{\mathrm{n}_{\mathrm{k}}}(\xi)$ is everywhere positive. Now re-index so the new sequence $\left\{f_{n}\right\}_{n}$ is the sequence $\left\{f_{n_{k}}\right\}_{k}$.

In addition it can be shown easily that there is a set $G$ of $\mathrm{x}$ with positive measure such that $Z^{1}(\mathrm{x}, 0) \neq 0$.

Since $\left.\hat{\mathrm{f}_{\mathrm{n}}}(\xi)\right|_{|\xi|>\delta} \in \mathrm{L}^{2}$ are uniformly $\mathrm{L}^{2}$-bounded in $\mathrm{n}$ for any fixed $\delta>0$ (as they have norm 1 in $H_{h}^{s}$ ),

$$
\lim _{n \rightarrow \infty} \int_{|\xi|<\delta} \hat{\psi}^{1}(\xi) \hat{f_{n}}(\xi) d \xi=\infty
$$

for any $\delta>0$. Also for any $\mathrm{x}$ and $\lambda$, the Zak transform $Z^{\lambda}(\mathrm{x}, \xi)$ is continuous in $\xi$ at $\xi=0$, since it is a Fourier series $\xi$ with coefficients in $\ell^{1}$ (since $\psi \in \mathrm{L}^{1} \cap[\mathrm{RB}]$ ).

Consider linear combinations

$$
\hat{\psi}_{\mathbf{c}}(\xi)=\sum_{\lambda} \mathrm{c}_{\lambda} \overline{\hat{\psi}^{\lambda}}(\xi)
$$

with $\mathbf{c}=\left(\mathrm{c}_{1}, \mathrm{c}_{2}, \ldots, \mathrm{c}_{\ell}\right)$. Define the vector function $\hat{\boldsymbol{\psi}}(\xi)=\left(\overline{\hat{\psi}^{1}}, \ldots, \overline{\hat{\psi}^{\ell}}\right)(\xi)$, and consider the integral $\int \hat{\psi}(\xi) \cdot Z(x, \xi) \hat{\mathrm{f}_{\mathrm{n}}}(\xi) \mathrm{d} \xi$, where the inner product in the integrand is between the vectors $\hat{\psi}(\xi)$ and $\boldsymbol{Z}(\mathrm{x}, \xi) \equiv\left(Z^{1}, \ldots, Z^{\ell}\right)(\mathrm{x}, \xi)$. 
It follows from Lemma 2.14 and equation (2.8) that the set of vectors $\mathbf{c}$ such that

$$
\limsup _{n \rightarrow \infty}\left|\int_{|\xi|<\delta} \hat{\psi}_{c}(\xi) f_{n}(\xi) d \xi\right| \neq \infty
$$

must all satisfy a linear relation of the form $\mathbf{v} \cdot \mathbf{c}=0$, where $\mathbf{v}$ is a nontrivial vector.

We can then show that for some $G \subset \mathbb{R}^{\mathrm{d}}$ of positive measure, $\mathrm{x} \in G$ implies $\mathbf{v} \cdot \boldsymbol{Z}(\mathrm{x}, 0)$ $\neq 0$. Thus for $\mathrm{x} \in G$

$$
\limsup _{n \rightarrow \infty}\left|\int \hat{f_{n}}(\xi) \sum_{\lambda} \overline{\psi^{\lambda}}(\xi) Z^{\lambda}(x, 0) d \xi\right|=\infty .
$$

It is easy to show there exists a set $G_{1} \subseteq G$ of positive measure such that for $\mathrm{x} \in G_{1}$, $|\mathbf{v} \cdot \boldsymbol{Z}(\mathrm{x}, 0)|>\gamma>0$ for some fixed $\gamma>0$.

Consider now $\mathbf{w}_{\mathrm{n}} \equiv\left(\mathrm{w}_{\mathrm{n}}^{1}, \mathrm{w}_{\mathrm{n}}^{2}, \ldots, \mathrm{w}_{\mathrm{n}}^{\ell}\right)$, with

$$
\mathrm{w}_{\mathrm{n}}^{\lambda} \equiv \int \hat{\hat{\psi^{\lambda}}}(\xi) \hat{\mathrm{f}_{\mathrm{n}}}(\xi) \mathrm{d} \xi
$$

We henceforth assume the vector $\mathbf{v}$ is constructed as in Lemma 2.14, as a limit point of the directions of the vectors $\mathbf{w}_{\mathrm{n}}$, i.e., of $\widetilde{\mathbf{w}}_{\mathrm{n}} \equiv \mathbf{w}_{\mathrm{n}} /\left|\mathbf{w}_{\mathrm{n}}\right|$. We claim for $\mathrm{x} \in G_{1}$, there is a subsequence $\left\{\mathrm{n}_{\mathrm{k}}\right\}$ such that

$$
\left|\mathbf{w}_{\mathrm{n}_{\mathrm{k}}} \cdot \boldsymbol{Z}(\mathrm{x}, 0)\right|>\gamma \mathrm{w}_{\mathrm{n}_{\mathrm{k}}}^{1}
$$

for $\gamma$ as above. Indeed, $\widetilde{\mathbf{w}_{\mathrm{n}_{\mathrm{k}}}} \underset{\mathrm{k} \rightarrow \infty}{\rightarrow} \mathbf{v}$ for an appropriate subsequence, since $\mathbf{v}$ is a limit point of $\widetilde{\mathbf{w}_{\mathrm{n}_{\mathrm{k}}}}$. Thus for large $\mathrm{k}$

$$
\left|\mathbf{w}_{\mathrm{n}_{\mathrm{k}}} \cdot \boldsymbol{Z}(\mathrm{x}, 0)\right|=\left|\widetilde{\mathbf{w}}_{\mathrm{n}_{\mathrm{k}}} \cdot \boldsymbol{Z}(\mathrm{x}, 0)\right|\left|\mathbf{w}_{\mathrm{n}_{\mathrm{k}}}\right|>\gamma\left|\mathbf{w}_{\mathrm{n}_{\mathrm{k}}}\right| \geq \gamma \mathbf{w}_{\mathrm{n}_{\mathrm{k}}}^{1}
$$

(recall that $w_{n_{k}}^{1}$ is positive by our assumptions). Equivalently for $x \in G_{1}$

$$
\left|\int \hat{\mathrm{f}}_{\mathrm{n}_{\mathrm{k}}}(\xi) \sum_{\lambda} \overline{\hat{\psi}^{\lambda}}(\xi) Z^{\lambda}(\mathrm{x}, 0) \mathrm{d} \xi\right|>\gamma \int \hat{\mathrm{f}}_{\mathrm{n}_{\mathrm{k}}}(\xi) \overline{\hat{\psi}^{1}}(\xi) \mathrm{d} \xi
$$

Consider now the full integral in (2.6) (now including the variation in $\xi$ ),

$$
\int \hat{\mathrm{f}}_{\mathrm{n}_{\mathrm{k}}}(\xi) \sum_{\lambda} \overline{\hat{\psi}^{\lambda}}(\xi) Z^{\lambda}(\mathrm{x},-\xi) \mathrm{d} \xi
$$

With some additional arguments it can be shown that (2.10) holds as well if the left side is replaced by (2.11), for $x$ in another set $G_{2}$ of positive measure.

Thus for $x \in G_{2}$

$$
\left|\int \hat{\mathrm{f}}_{\mathrm{n}_{\mathrm{k}}}(\xi) \sum_{\lambda} \overline{\hat{\psi}^{\lambda}}(\xi) Z^{\lambda}(\mathrm{x},-\xi) \mathrm{d} \xi\right| \underset{\mathrm{k} \rightarrow \infty}{\rightarrow} \infty
$$

Thus by (2.6) 


$$
\left\|\int \mathrm{Q}_{0}(\mathrm{x}, \mathrm{y}) \mathrm{f}_{\mathrm{n}_{\mathrm{k}}}(\mathrm{y})\right\|_{\infty}=\left\|\int \hat{\mathrm{f}}_{\mathrm{n}_{\mathrm{k}}}(\xi) \sum_{\lambda} \overline{\hat{\psi}^{\lambda}}(\xi) Z^{\lambda}(\mathrm{x},-\xi) \mathrm{d} \xi\right\|_{\infty} \underset{\mathrm{k} \rightarrow \infty}{\rightarrow} \infty
$$

so that $\left\|\mathrm{Q}_{0}\right\| \mathrm{H}_{\mathrm{h}}^{\mathrm{s}} \rightarrow L^{\infty}=\infty$, since $\left\|\mathrm{f}_{\mathrm{n}_{\mathrm{k}}}\right\|_{\mathrm{H}_{\mathrm{h}}^{\mathrm{s}}}=1$ for all $\mathrm{n}_{\mathrm{k}}$. By scaling therefore for all $\mathrm{n}$,

$$
\left\|\mathrm{Q}_{\mathrm{n}}\right\|_{\mathrm{H}_{\mathrm{h}}^{\mathrm{s}} \rightarrow L^{\infty}}=\infty \text {. }
$$

Now consider the projection $\mathrm{P}_{1}=\mathrm{P}_{0}+\mathrm{Q}_{0}$. We have

$$
\left\|\left(\mathrm{P}_{1}-\mathrm{I}\right) \mathrm{f}_{\mathrm{n}_{\mathrm{k}}}\right\|_{\infty}=\left\|\left(\mathrm{P}_{0}-\mathrm{I}\right) \mathrm{f}_{\mathrm{n}_{\mathrm{k}}}+\mathrm{Q}_{0} \mathrm{f}_{\mathrm{n}_{\mathrm{k}}}\right\|_{\infty},
$$

so that if $\left\|\left(\mathrm{P}_{0}-\mathrm{I}\right) \mathrm{f}_{\mathrm{n}_{\mathrm{k}}}\right\| \infty$ is bounded in $\mathrm{k}$, then by the above equation $\left\|\left(\mathrm{P}_{1}-\mathrm{I}\right) \mathrm{f}_{\mathrm{n}_{\mathrm{k}}}\right\| \infty$ is unbounded in $\mathrm{k}$. Thus by scaling invariance $\left\|\left(\mathrm{P}_{0}-\mathrm{I}\right)\right\| \mathrm{H}_{\mathrm{h}}^{\mathrm{s}} \rightarrow \mathrm{L}^{\infty}=\infty$ (since the two norms are infinite or finite together). This completes the proof.

Combining Theorems 2.8 and 2.15:

Corollary $2.16\left((\mathrm{a}) \Leftrightarrow\left(\mathrm{c}^{\prime}\right)\right.$ in Theorem 1): For $s>0$ a necessary and sufficient condition for order $s-d / 2$ convergence in $H^{s}$ of wavelet expansions with basic wavelets $\psi$ satisfying $\psi^{\lambda}(x) \in[\mathrm{RB}]$ is that for all $\lambda$

$$
\int_{|\xi|<\delta}\left|\hat{\psi^{\lambda}}(\xi)\right|^{2}|\xi|^{-2 s} \mathrm{~d} \xi<\infty
$$

for some (or for all) $\delta>0$ (including $\delta=\infty)$.

This follows because for $\psi \in \mathrm{L}^{2}$, (2.12) states that $\psi \in \mathrm{H}_{\mathrm{h}}^{-\mathrm{s}}$.

Remarks: 1. Note this result is intuitively expected for s close to 0 , since then order $s$ convergence naturally requires $\mathrm{f} \in \mathrm{H}^{\mathrm{s}+\mathrm{d} / 2}$, since otherwise $f$ need not even be continuous.

2. Order $s$ convergence in this Theorem (and the other parts of Theorem 1) applies to any summation order in which scale $\mathrm{j}$ wavelets are added before scale $\mathrm{j}+1$ wavelets. This is best seen from the fact that not only $\mathrm{I}-\mathrm{P}_{\mathrm{j}}$ satisfies the bounds in the proof of Theorem 2.15, but also any part $\mathrm{Q}_{\mathrm{jK}}(\mathrm{x}, \mathrm{y})=\sum_{\mathrm{k} \in \mathrm{K}} \psi_{\mathrm{jk}}(\mathrm{x}) \overline{\psi_{\mathrm{jk}}}(\mathrm{y})$ of $\mathrm{Q}_{\mathrm{j}}$. Precisely, we have that the norm $\mathrm{Q}_{0 \mathrm{~K}}: \mathrm{H}_{\mathrm{h}}^{\mathrm{s}} \rightarrow \mathrm{L}^{\infty}$ is bounded uniformly in the choice of the set $\mathrm{K}$, using arguments identical to those for I - $\mathrm{P}_{\mathrm{j}}$ in the first part of the proof (sufficiency) of Theorem 2.15.

This statement easily extends to the statement that the present results hold for any order of summation in which the range of values of $\mathrm{j}$ for which the sum over $\mathrm{k}$ and $\lambda$ is partially complete always remains bounded.

\section{Proofs of conditions on scaling functions}

We now translate condition (2.12) into one on the scaling function $\phi$. First under our hypotheses, 


$$
\int_{\mathbb{R}^{\mathrm{d}}} \phi(\mathrm{x}) \mathrm{dx}=1
$$

if $\phi$ is multiplied by a possible phase constant. To see this note that under the assumption $\phi, \psi \in[\mathrm{RB}]$, the function $s(x) \equiv \sum_{\mathrm{k} \in \mathbb{Z}^{d}} \phi(\mathrm{x}-\mathrm{k})$ is uniformly bounded in $\mathrm{x}$ through an argument using the radial bound $\eta(\mathrm{x})$ for $\phi(\mathrm{x})$, and the fact that the sum can be bounded by a constant times $\int \eta(\mathrm{x}-\mathrm{y}) \mathrm{dy}<\infty$. Thus by dominated convergence for $\ell \in \mathbb{Z}^{d}$

$$
\int_{\mathbb{R}^{\mathrm{d}}} \bar{\phi}(\mathrm{x}-\ell) \mathrm{s}(\mathrm{x}) \mathrm{dx}=\sum_{\mathrm{k} \in \mathbb{Z}^{\mathrm{d}}} \int_{\mathbb{R}^{\mathrm{d}}} \bar{\phi}(\mathrm{x}) \phi(\mathrm{x}-\mathrm{k}) \mathrm{dx}=1 .
$$

Similarly for any $\psi_{\mathrm{jk}}$ with $j \geq 0$

$$
\int_{\mathbb{R}^{\mathrm{d}}} \overline{\psi_{\mathrm{jk}}}(\mathrm{x}) \mathrm{s}(\mathrm{x}) \mathrm{dx}=0
$$

It is not hard to show that $\mathrm{s} \in \mathrm{L}^{\infty}$ is uniquely identified by (3.2) and (3.3). Thus

$$
\mathrm{s}(\mathrm{x})=\sum_{\mathrm{k}} \phi(\mathrm{x}-\mathrm{k}) \equiv \frac{1}{\int_{\mathbb{R}^{d}} \bar{\phi}(y) \mathrm{d} y}
$$

since this satisfies $(3.2,3.3)$. Also by dominated convergence

$$
\int \bar{\phi}(\mathrm{x}) \mathrm{dx}=\sum_{\mathrm{k} \in \mathbb{Z}^{\mathrm{d}}} \int_{\mathrm{C}} \bar{\phi}(\mathrm{x}-\mathrm{k}) \mathrm{dx}=\int_{\mathrm{C}} \sum_{\mathrm{k} \in \mathbb{Z}^{\mathrm{d}}} \bar{\phi}(\mathrm{x}-\mathrm{k}) \mathrm{dx},
$$

with $\mathrm{C}$ the unit cube $\left\{\mathrm{x} \in \mathbb{R}^{\mathrm{d}} \mid 0 \leq \mathrm{x}_{\mathrm{i}} \leq 1\right\}$. By this and the complex conjugate of (3.4)

$$
\int \bar{\phi}(\mathrm{x}) \mathrm{dx}=\int_{\mathrm{C}} \frac{1}{\int \phi(\mathrm{y}) \mathrm{dy}} \mathrm{dx}=\frac{1}{\int \phi(\mathrm{y}) \mathrm{dy}},
$$

so $\left|\int \phi(x) d x\right|^{2}=1$, and after possible multiplication of $\phi$ by a phase (3.1) holds, and

$$
\hat{\phi}(0)=\frac{1}{(2 \pi)^{\mathrm{d} / 2}} .
$$

Let $F=\{0,1\}^{\mathrm{d}}$ be all vectors with entries consisting of $\{0,1\}$. The following is an extension of a standard one dimensional fact:

Lemma 3.1: If $\phi$ is a scaling function for a multiresolution analysis, then

$$
\sum_{\ell \in \mathbb{Z}^{d}}|\hat{\phi}(\xi+2 \pi \ell)|^{2}=(2 \pi)^{-d}
$$

Furthermore for any $\epsilon \in F$, 


$$
\sum_{\ell \in 2 \mathbb{Z}^{\mathrm{d}}+\epsilon}|\hat{\phi}(\xi+\pi \ell)|^{2}=(2 \pi)^{-\mathrm{d}}
$$

Thus:

Lemma 3.2: If $\phi$ is a scaling function for a multiresolution analysis, then $\hat{\phi}(0)=(2 \pi)^{-\mathrm{d} / 2}$ and $\hat{\phi}(2 \pi \ell)=0$ for $\ell \neq 0$.

Proof: This follows from (3.5), and Lemma 3.1.

Corollary 3.3: If $\phi$ is a scaling function from a multiresolution analysis

$$
\sum_{\ell \in \mathbb{Z}^{d} ; \ell \neq 0}|\hat{\phi}(\xi+2 \pi \ell)|^{2} \leq C\left((2 \pi)^{-d / 2}-|\phi(\xi)|\right)
$$

for some $C>0$.

Proof: By Lemma 3.1

$$
\begin{aligned}
\sum_{\ell \in \mathbb{Z}^{\mathrm{d}} ; \ell \neq 0}|\hat{\phi}(\xi+2 \pi \ell)|^{2}=(2 \pi)^{-\mathrm{d}} & -|\widehat{\phi}(\xi)|^{2} . \\
& =\left((2 \pi)^{-d / 2}+|\widehat{\phi}(\xi)|\right)\left((2 \pi)^{-d / 2}-|\phi(\xi)|\right) \\
& \leq C\left((2 \pi)^{-d / 2}-|\widehat{\phi}(\xi)|\right)
\end{aligned}
$$

since $\widehat{\phi}$ is bounded.

If $\phi$ is a scaling function for a multiresolution analysis, the coefficients $\left\{h_{\mathrm{k}}\right\}_{\mathrm{k} \in \mathbb{Z}^{\mathrm{d}}}$ are defined by

$$
\phi(\mathrm{x})=2^{\mathrm{d}} \sum_{\mathrm{k} \in \mathbb{Z}^{\mathrm{d}}} h_{\mathrm{k}} \phi(2 \mathrm{x}-\mathrm{k})
$$

Fourier transforming (3.6)

$$
\hat{\phi}(\xi)=\sum_{\mathrm{k} \in \mathbb{Z}^{\mathrm{d}}} h_{\mathrm{k}} \mathrm{e}^{-\mathrm{ik} \xi / 2} \hat{\phi}(\xi / 2)=\mathrm{m}_{0}(\xi / 2) \hat{\phi}(\xi / 2)
$$

Thus

$$
\mathrm{m}_{0}(0)=1
$$

Then 
yielding

$$
\begin{aligned}
(2 \pi)^{-\mathrm{d}}=\sum_{\ell \in \mathbb{Z}^{\mathrm{d}}} & |\hat{\phi}(\xi+2 \pi \ell)|^{2} \\
& =\sum_{\ell \in \mathbb{Z}^{\mathrm{d}}}\left|\mathrm{m}_{0}(\xi / 2+\pi \ell)\right|^{2}|\hat{\phi}(\xi / 2+\pi \ell)|^{2} \\
& =\sum_{\epsilon \in F} \sum_{\ell \in 2 \mathbb{Z}^{\mathrm{d}}+\epsilon}\left|\mathrm{m}_{0}(\xi / 2+\pi \epsilon)\right|^{2}|\hat{\phi}(\xi / 2+\pi \ell)|^{2} . \\
& =(2 \pi)^{-\mathrm{d}} \sum_{\epsilon \in F}\left|\mathrm{~m}_{0}(\xi / 2+\pi \epsilon)\right|^{2},
\end{aligned}
$$

$$
\sum_{\epsilon \in F}\left|\mathrm{~m}_{0}(\xi+\pi \epsilon)\right|^{2}=1
$$

a.e. for $\xi \in \mathbb{R}^{\mathrm{d}}$. Together with (3.8), this shows $\mathrm{m}_{0}(\xi)$ assumes its maximum value of 1 at $\xi$ $=0$. In the third equality above we have used the $2 \pi$ - periodicity of $\mathrm{m}_{0}$ in all coordinate directions, and in the last we have used Lemma 3.1

For the following theorem we need a general version of the Poisson summation formula [SW, Theorem VII.2.4]. Given $\mathrm{f}(\mathrm{x}) \in \mathrm{L}^{1}\left(\mathbb{R}^{\mathrm{d}}\right)$ (using our Fourier transform conventions)

$$
\sum_{k \in \mathbb{Z}^{d}} f(x+k)=(2 \pi)^{d / 2} \sum_{l \in \mathbb{Z}^{d}} \hat{f}(2 \pi l) e^{2 \pi i x l}
$$

with $\hat{f}$ the Fourier transform of $f$.

Proposition 3.4: For $s \in \mathbb{R}$, an operator $R: \mathrm{H}_{\mathrm{h}}^{\mathrm{s}} \rightarrow \mathrm{L}^{\infty}$ with kernel $R(x, y)$ is bounded if and only if $R(\mathrm{x}, \mathrm{y}) \in \mathrm{H}_{\mathrm{h}}^{-\mathrm{s}}$ in the variable $y$, uniformly in $x$, i.e.,

$$
\int\left|\widehat{R}^{2}(x, \xi)\right||\xi|^{-2 s} d \xi<C<\infty
$$

for almost all $x$, where $\widehat{R}(x, \xi)$ is the Fourier transform of $R$ in $y$.

Proof: Assume first $\mathrm{R}: \mathrm{H}_{\mathrm{h}}^{\mathrm{s}} \rightarrow \mathrm{L}^{\infty}$ is bounded. To show $\int|\hat{\mathrm{R}}(\mathrm{x}, \xi)|^{2}|\xi|^{-2 s} \mathrm{~d} \xi$ is uniformly bounded in $\mathrm{x}$, we have

$$
\begin{aligned}
& \|\mathrm{R}\| \mathrm{H}_{\mathrm{h}}^{\mathrm{s}} \rightarrow \mathrm{L}^{\infty}=\operatorname{ess} \sup _{\mathrm{X}} \sup _{\|\mathrm{f}\| \|_{\mathrm{h}, \mathrm{s}}=1}|\operatorname{Rf}(\mathrm{x})| \\
& =\operatorname{ess} \sup _{\mathrm{X}}\left(\int|\hat{\mathrm{R}}(\mathrm{x}, \xi)|^{2}|\xi|^{-2 \mathrm{~s}} \mathrm{~d} \xi\right)^{1 / 2}
\end{aligned}
$$

since in the norm of a function (in this case $\widehat{R}(x, \xi)$ viewed as a function of $\xi$ ) is the supremum of its values on functionals in the unit ball of the dual space.

Conversely if $\hat{R}(x, y) \in \mathrm{H}_{h}^{-s}$ essentially uniformly in $x$, then $\mathrm{R}: \mathrm{H}_{\mathrm{h}}^{\mathrm{s}} \rightarrow \mathrm{L}^{\infty}$ is bounded by the Schwartz inequality:

$$
\left\|\int \hat{\mathrm{R}}(\mathrm{x}, \xi) \hat{\mathrm{f}}(-\xi) \mathrm{d} \xi\right\|_{\infty}^{2} \leq\left\|\int|\hat{\mathrm{R}}(\mathrm{x}, \xi)|^{2}|\xi|^{-2 \mathrm{~s}} \mathrm{~d} \xi\right\|_{\infty} \int|\hat{\mathrm{f}}(\xi)|^{2}|\xi|^{2 \mathrm{~s}} \mathrm{~d} \xi
$$




$$
=\left\|\int|\hat{\mathrm{R}}(\mathrm{x}, \xi)|^{2}|\xi|^{-2 \mathrm{~s}} \mathrm{~d} \xi\right\|_{\infty}\|\mathrm{f}\|_{\mathrm{h}, \mathrm{s}}^{2} .
$$

Recall $E=E_{0}=I-P$.

Corollary 3.5: The MRA $\left\{P_{n}\right\}$ has order of approximation $s-d / 2$ in $H^{\mathrm{s}}$ if and only if the error $E(\mathrm{x}, y) \equiv \mathcal{F}_{\xi}(\widetilde{E}(x, \xi)) \in \mathrm{H}_{\mathrm{h}}^{-\mathrm{s}}$ in the variable y, uniformly in $x$.

Proof: This is an immediate corollary of Proposition 3.4 and Theorem 1.

Equivalently (recall $\tilde{E}(x, \xi)$ is defined before Proposition 2.6):

Corollary 3.6: The MRA $\left\{P_{n}\right\}$ has order of approximation $s-d / 2$ in $H^{\mathrm{s}}$ if and only if $\int|\tilde{E}(\mathrm{x}, \xi)|^{2}|\xi|^{-2 \mathrm{~s}} \mathrm{~d} \xi$ is essentially bounded in $x$.

We remark that the following theorem has at least two proofs, one taking advantage of relationships between the Fourier transforms of the scaling function $\phi$ and wavelets $\psi^{\lambda}$ (with the additional assumption that the latter are radially bounded). Both proofs have approximately equal complexity, and we include that which does not rely on existence of wavelets.

Theorem 3.7 ((a) $\Leftrightarrow$ (d) in Theorem 1): Let $\phi$ be the scaling function of a multiresolution analysis, and assume $\phi \in[\mathrm{RB}], s>\mathrm{d} / 2$. A necessary and sufficient condition for order $s-d / 2$ convergence in $H^{s}$ of the multiresolution approximation with scaling function $\phi$ is that $\phi$ satisfy

$$
\int_{|\xi|<\delta}\left(1-(2 \pi)^{\mathrm{d} / 2}|\hat{\phi}(\xi)|\right)|\xi|^{-2 \mathrm{~s}} \mathrm{~d} \xi<\infty
$$

for some (or for all) $\delta>0$.

Proof: Assume first that (3.11) is satisfied. We only sketch a proof of the implication that we have order $s-d / 2$ convergence (see remark at end of Section 1). By Theorem 2.8 we need to show $E_{0} \equiv \mathrm{I}-\mathrm{P}_{0}$ is bounded from $\mathrm{H}_{\mathrm{h}}^{\mathrm{s}}$ to $\mathrm{L}^{\infty}$. By Corollary 3.6, it suffices to show $\int d \xi\left|\tilde{E}_{0}(x, \xi)\right|^{2}|\xi|^{-2 s}$ is essentially bounded in $x$. Since $E_{0}=I-P_{0}$, we consider the kernel of $P_{0}$; recall that the kernel in Fourier space $\hat{P}_{0}(x, \xi)=\widetilde{P}_{0}(x,-\xi)$ is the Fourier transform of $P(x, y)$ in $y$ :

$$
\begin{aligned}
& \widehat{P}_{0}(x, \xi) \equiv \mathcal{F}_{y}\left(P_{0}(x, y)\right)=\mathcal{F}_{y}\left(\sum_{k} \phi(x-k) \bar{\phi}(y-k)\right) \\
& =\sum_{\mathrm{k}} \phi(\mathrm{x}-\mathrm{k})(2 \pi)^{-d / 2} \overline{\int \phi(\mathrm{y}) \mathrm{e}^{\mathrm{i}(\mathrm{y}+\mathrm{k}) \xi} \mathrm{dy}} \\
& =Z(\mathrm{x}, \xi) \overline{\hat{\phi}}(-\xi),
\end{aligned}
$$


where $Z(\mathrm{x}, \xi) \equiv \sum_{\mathrm{k}} \phi(\mathrm{x}-\mathrm{k}) \mathrm{e}^{-\mathrm{i} \xi \mathrm{k}}=\sum_{\mathrm{k}} \phi(\mathrm{x}+\mathrm{k}) \mathrm{e}^{\mathrm{i} \xi \mathrm{k}}$ is the Zak transform of $\phi$. Thus $\tilde{E}_{0}(x, \xi)$, the kernel of $E_{0}$ in Fourier space, is ( $I$ denotes the identity)

$$
\widetilde{E}_{0}(x, \xi)=\widetilde{I}(x, \xi)-\widehat{P}_{0}(x,-\xi)=(2 \pi)^{-d / 2} e^{i x \xi}-Z(x,-\xi \widehat{\widehat{\phi}}(\xi)
$$

Define $\alpha(\mathrm{x}) \equiv \phi(\mathrm{x}) \mathrm{e}^{\mathrm{i} \xi \mathrm{x}}$, so $\hat{\alpha}(\mathrm{w})=\hat{\phi}(\mathrm{w}-\xi)$. Using the Poisson formula and recalling $F=\{0,1\}^{d}$, it can be shown

$$
\begin{aligned}
& Z(\mathrm{x}, \xi) \equiv \sum_{\mathrm{k} \in \mathbb{Z}^{\mathrm{d}}} \phi(\mathrm{x}+\mathrm{k}) \mathrm{e}^{\mathrm{i} \xi \mathrm{k}} \\
& \quad=(2 \pi)^{\mathrm{d} / 2} \mathrm{e}^{-\mathrm{i} \xi \mathrm{x}} \sum_{\epsilon \in F} \mathrm{~m}_{0}(\pi \epsilon-\xi / 2) \sum_{l \in 2 \mathbb{Z}^{\mathrm{d}}+\epsilon} \hat{\phi}(\pi l-\xi / 2) \mathrm{e}^{2 \pi \mathrm{ix} l} .
\end{aligned}
$$

Note that $Z(x, \xi)$ is continuous in $\xi$ for each $x$, since its Fourier series has coefficients $\phi(x+k)$ in $\ell^{1}$. Further, the functions $Z(x, \xi)$ are easily shown to be uniformly continuous in $\xi$, in the parameter $x$, since $\phi \in[\mathrm{RB}]$.

Another calculation then shows

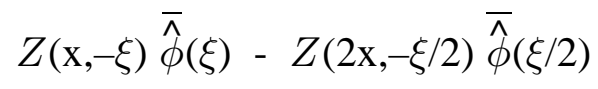

$$
\begin{aligned}
&=\left(\left|\mathrm{m}_{0}(\xi / 2)\right|^{2}-1\right) \overline{\hat{\phi}}(\xi / 2) Z(2 \mathrm{x},-\xi / 2) \\
&+\overline{\mathrm{m}_{0}}(\xi / 2) \overline{\hat{\phi}}(\xi / 2) \sum_{\epsilon \neq 0} \mathrm{~m}_{0}(\pi \epsilon+\xi / 2) Z(2 \mathrm{x},-\xi / 2-\pi \epsilon)
\end{aligned}
$$

Some additional calculations using the relationship of $m_{0}(\xi)$ and $\phi(\xi)$ show

$$
\int_{|\xi|<\delta}\left(\left|1-\mathrm{m}_{0}(\xi / 2)\right|^{2}\right)|\xi|^{-2 s} \mathrm{~d} \xi<\infty .
$$

Using (3.9) we can then show

$$
\int\left|\mathrm{m}_{0}(\pi \epsilon+\xi / 2)\right|^{2}|\xi|^{-2 \mathrm{~s}} \mathrm{~d} \xi<\infty .
$$

Combining (3.13), (3.14), and (3.15) ( $\mathrm{L}^{\infty}$ norms are in $\mathrm{x}$ only), a calculation gives

$$
\begin{aligned}
& \left(\left.\int_{|\xi|<\delta}\left|\left\|e^{-i x \xi} Z(\mathrm{x},-\xi) \hat{\phi}(\xi)-(2 \pi)^{d / 2}\right\| \infty^{-}\left\|e^{-i x \xi} Z(2 \mathrm{x},-\xi / 2) \overline{\hat{\phi}}(\xi / 2)-(2 \pi)^{d / 2}\right\| \|_{\infty}\right| \xi\right|^{-2 \mathrm{~s}} \mathrm{~d} \xi\right)^{1 / 2} \\
& \leq\left(\int_{|\xi|<\delta}\left|\left(\left|\mathrm{m}_{0}(\xi / 2)\right|^{2}-1\right) \hat{\phi}(\xi / 2)\right|\|Z(2 \mathrm{x},-\xi / 2)\| \|_{\infty}^{2}|\xi|^{-2 \mathrm{~s}} \mathrm{~d} \xi\right)^{1 / 2} \\
& +\left(\left.\int_{|\xi|<\delta}\left|\overline{\mathrm{m}}_{0}(\xi / 2) \hat{\hat{\phi}}(\xi / 2)\right| \sum_{\epsilon \neq 0}\left|\mathrm{~m}_{0}(\pi \epsilon+\xi / 2)\right|\|Z(2 \mathrm{x},-\xi / 2-\pi \epsilon)\|_{\infty}\right|^{2}|\xi|^{-2 \mathrm{~s}} \mathrm{~d} \xi\right)^{1 / 2} \\
& <\infty
\end{aligned}
$$

since $Z(\mathrm{x}, \xi)$ is uniformly bounded in $\mathrm{x}$ and $\xi$, as are $\widehat{\phi}$ and $m_{0}$.

Defining 


$$
\mathrm{F}(\xi) \equiv\left\|\left(\mathrm{e}^{-\mathrm{ix} \xi} Z(\mathrm{x},-\xi) \hat{\hat{\phi}}(\xi)-(2 \pi)^{-\mathrm{d} / 2}\right)|\xi|^{-\mathrm{s}}\right\|_{\infty}
$$

we remark for future reference (part II of this paper) that this function is continuous in $\xi$, being the supremum in $x$ of a collection of functions which are uniformly continuous in $\xi$ (see remark before (3.13)).

We have by the above

$$
\int_{|\xi|<\delta}\left|\mathrm{F}(\xi)-2^{-\mathrm{s}} \mathrm{F}(\xi / 2)\right|^{2} \mathrm{~d} \xi<\infty .
$$

Since $Z(\mathrm{x}, \xi)$ and $\hat{\phi}(\xi)$ are both uniformly bounded in $\mathrm{x}$ and $\xi$ and $s>d / 2$, it follows in fact that

$$
B^{2} \equiv \int_{\mathbb{R}^{\mathrm{d}}}\left|\mathrm{F}(\xi)-2^{-\mathrm{s}} \mathrm{F}(\xi / 2)\right|^{2} \mathrm{~d} \xi<\infty
$$

Through some technical arguments we can then show from this that $F \in L^{2}\left(\mathbb{R}^{d}\right)$. Thus

$$
\int\left\|\mathrm{e}^{-\mathrm{i} \xi \mathrm{x}} Z(\mathrm{x},-\xi) \hat{\phi}(\xi)-(2 \pi)^{-\mathrm{d} / 2}\right\|_{\infty}^{2}|\xi|^{-2 \mathrm{~s}} \mathrm{~d} \xi<\infty .
$$

Thus by Corollary 3.6 $E_{0}: H_{h}^{s} \rightarrow L^{\infty}$ is bounded, and thus by Theorem 2.9 the multiresolution expansion with scaling function $\phi$ has order of convergence $s-d / 2$.

To prove the converse, assume

$$
\int_{|\xi|<\delta}\left(1-(2 \pi)^{\mathrm{d} / 2}|\hat{\phi}(\xi)|\right)|\xi|^{-2 \mathrm{~s}} \mathrm{~d} \xi=\infty .
$$

By (3.12)

$$
\begin{array}{r}
(2 \pi)^{-\mathrm{d} / 2} \mathrm{e}^{\mathrm{ix} \xi}-Z(\mathrm{x},-\xi) \hat{\hat{\phi}}(\xi) \\
=(2 \pi)^{-\mathrm{d} / 2} \mathrm{e}^{\mathrm{ix} \xi}\left(1-\sum_{l \in \mathbb{Z}^{\mathrm{d}}}(2 \pi)^{\mathrm{d}} \hat{\phi}(2 \pi l+\xi) \mathrm{e}^{2 \pi \mathrm{ixl} l} \overline{\hat{\phi}}(\xi)\right) .
\end{array}
$$

The second factor is

$$
\begin{aligned}
& 1-\sum_{l \in \mathbb{Z}^{\mathrm{d}}}(2 \pi)^{\mathrm{d}} \hat{\phi}(2 \pi l+\xi) \mathrm{e}^{2 \pi \mathrm{ix} l} \overline{\hat{\phi}}(\xi)= \\
&=\left(1-(2 \pi)^{\mathrm{d}}|\overline{\hat{\phi}}(\xi)|^{2}\right)-\sum_{l \neq 0}(2 \pi)^{\mathrm{d}} \hat{\phi}(\xi) \hat{\phi}(2 \pi l+\xi) \mathrm{e}^{2 \pi \mathrm{ix} l} .
\end{aligned}
$$

Letting $C$ denote the unit cube in $\mathbb{R}^{d}$, 


$$
\begin{aligned}
& \int_{C} d x \int_{|\xi|<\delta}\left|(2 \pi)^{-\mathrm{d} / 2}\left(1-(2 \pi)^{\mathrm{d}}|\hat{\phi}(\xi)|^{2}\right)-(2 \pi)^{\mathrm{d} / 2} \sum_{l \neq 0} \overline{\hat{\phi}}(\xi) \hat{\phi}(2 \pi l+\xi) \mathrm{e}^{2 \pi \mathrm{i} x l}\right|^{2}|\xi|^{-2 s} \mathrm{~d} \xi \\
& =(2 \pi)^{d} \int_{|\xi|<\delta}\left|(2 \pi)^{-\mathrm{d} / 2}\left(1-(2 \pi)^{\mathrm{d}}|\hat{\hat{\phi}}(\xi)|^{2}\right)\right|^{2}+\sum_{l \neq 0}\left|(2 \pi)^{\mathrm{d} / 2} \overline{\hat{\phi}}(\xi) \hat{\phi}(2 \pi l+\xi)\right|^{2}|\xi|^{-2 s} \mathrm{~d} \xi \\
& \geq(2 \pi)^{d} \int_{|\xi|<\delta} \sum_{l \neq 0}\left|(2 \pi)^{\mathrm{d} / 2} \overline{\hat{\phi}}(\xi) \hat{\phi}(2 \pi l+\xi)\right|^{2}|\xi|^{-2 s} \mathrm{~d} \xi
\end{aligned}
$$

Note the first equality follows from the Parseval equality for Fourier series, since the $x$ integration (once the $x$ and $\xi$ integrations are interchanged) is the square of the $L^{2}$-norm of a Fourier series in $x$.

On the other hand by (3.17), factoring the difference of squares below and using Lemma 3.1,

$$
(2 \pi)^{d} \int_{|\xi|<\delta}\left(\sum_{\ell \neq 0}|\widehat{\phi}(\xi+2 \pi \ell)|^{2}\right)|\xi|^{-2 s} \mathrm{~d} \xi=\int_{|\xi|<\delta}\left(1-(2 \pi)^{\mathrm{d}}|\hat{\phi}(\xi)|^{2}\right)|\xi|^{-2 s} \mathrm{~d} \xi=\infty,
$$

so comparing with (3.18), (since $\widehat{\phi}(0) \neq 0$ and $\widehat{\phi}$ is continuous)

$$
\int_{C} d x \int_{|\xi|<\delta}\left|(2 \pi)^{-\mathrm{d} / 2} e^{i x \xi}-Z(\mathrm{x},-\xi) \overline{\hat{\phi}}(\xi)\right|^{2}|\xi|^{-2 s} \mathrm{~d} \xi=\infty .
$$

The above is an $L^{1}$ norm over the unit cube $C$ in $x$ of a periodic function in $x$, and so the $L^{\infty}$ norm of the function is also infinite.

Therefore the pseudodifferential operator

$$
\tilde{E}_{0}(x, \xi)=(2 \pi)^{-\mathrm{d} / 2} e^{i x \xi}-Z(\mathrm{x},-\xi) \overline{\hat{\phi}}(\xi)
$$

satisfies

$$
\left\|\int\left|\tilde{E}_{0}(x, \xi)\right|^{2}|\xi|^{-2 s} d \xi\right\|_{\infty}=\infty
$$

Applying proposition 3.4, we conclude the corresponding operator $E_{0}: H_{h}^{s} \rightarrow L^{\infty}$ is unbounded, so by Theorem 1, we do not have order $s-d / 2$ convergence in $H^{s}$.

Theorem $3.8((\mathrm{~d}) \Leftrightarrow(\mathrm{e}))$ : For real $t$ and $\delta>0$,

$$
\int_{|\xi|<\delta}\left(1-(2 \pi)^{\mathrm{d} / 2}|\hat{\phi}(\xi)|\right)|\xi|^{-\mathrm{t}} \mathrm{d} \xi<\infty
$$

if and only if 


$$
\int_{|\xi|<\delta}\left(1-\left|\mathrm{m}_{0}(\xi)\right|^{2}\right)|\xi|^{-\mathrm{t}} \mathrm{d} \xi<\infty
$$

Proof: Assume the first condition above. Then (see (3.7))

$$
\left|\mathrm{m}_{0}(\xi)\right|^{2}-1=\left((2 \pi)^{\mathrm{d}}|\widehat{\phi}(2 \xi)|^{2}-1\right)-\left|\mathrm{m}_{0}(\xi)\right|^{2}\left((2 \pi)^{\mathrm{d}}|\widehat{\phi}(\xi)|^{2}-1\right),
$$

which immediately yields the second condition (upon factoring the differences of squares on the right of (3.21)).

Conversely assume the second condition in the statement holds. We have

$$
\begin{gathered}
\left(1-(2 \pi)^{\mathrm{d}}|\widehat{\phi}(2 \xi)|^{2}\right)-\left(1-(2 \pi)^{\mathrm{d}}|\widehat{\phi}(\xi)|^{2}\right)=(2 \pi)^{\mathrm{d}}\left(|\widehat{\phi}(\xi)|^{2}-|\widehat{\phi}(2 \xi)|^{2}\right) \\
=(2 \pi)^{\mathrm{d}}\left(|\widehat{\phi}(\xi)|^{2}-\left|\mathrm{m}_{0}(\xi) \widehat{\phi}(\xi)\right|^{2}\right) \\
=(2 \pi)^{\mathrm{d}}\left(1-\left|\mathrm{m}_{0}(\xi)\right|^{2}\right)|\widehat{\phi}(\xi)|^{2} .
\end{gathered}
$$

The factors in the integrand of (3.20) are positive since $m_{0}(\xi)$ assumes its maximum value of 1 at $\xi=0$, as shown earlier. Thus

$$
\int_{|\xi|<\delta}\left|\left(1-(2 \pi)^{\mathrm{d}}|\hat{\phi}(\xi)|^{2}\right)-\left(1-(2 \pi)^{\mathrm{d}}|\hat{\phi}(\xi / 2)|^{2}\right)\right||\xi|^{\mathrm{t}} \mathrm{d} \xi<\infty .
$$

Defining $\mathrm{F}(\xi) \equiv\left(1-(2 \pi)^{\mathrm{d}}|\hat{\phi}(\xi)|^{2}\right)|\xi|^{-\mathrm{t}} \mathrm{d} \xi$,

$$
\int_{|\xi|<\delta}\left|\mathrm{F}(\xi)-2^{-\mathrm{t}} \mathrm{F}(\xi / 2)\right| \mathrm{d} \xi<\infty .
$$

Proceeding now as in (3.16), we define $\mathrm{G}(\xi) \equiv \mathrm{F}(\xi) \chi_{\{|\xi| \leq 2 \delta\}}(\xi)$, and get:

$$
\int\left|\mathrm{G}(\xi)-2^{-\mathrm{t}} \mathrm{G}(\xi / 2)\right| \mathrm{d} \xi<\infty .
$$

Let $\mathrm{G}_{\mathrm{n}}(\xi)$ be $\mathrm{L}^{1}$ functions which increase monotonically pointwise to $\mathrm{G}$, such that

$$
\left|\mathrm{G}_{\mathrm{n}}(\xi)-2^{-\mathrm{t}} \mathrm{G}_{\mathrm{n}}(\xi / 2)\right| \leq\left|\mathrm{G}(\xi)-2^{-\mathrm{t}} \mathrm{G}(\xi / 2)\right| ;
$$

these can be constructed with some simple arguments.

Now we have:

$$
\begin{gathered}
\left\|\mathrm{G}_{\mathrm{n}}(\xi)-2^{-\mathrm{t}} \mathrm{G}_{\mathrm{n}}(\xi / 2)\right\|_{1} \geq\left\|\mathrm{G}_{\mathrm{n}}(\xi)\right\|_{1}-\left\|2^{-\mathrm{t}} \mathrm{G}_{\mathrm{n}}(\xi / 2)\right\|_{1} \\
=\left(1-2^{\mathrm{d}-\mathrm{t}}\right)\left\|\mathrm{G}_{\mathrm{n}}(\xi)\right\|_{1} .
\end{gathered}
$$

Since the left side of (3.23) is bounded uniformly in $\mathrm{n}$ by dominated convergence, it follows the same holds for the right side, and again by dominated convergence $\mathrm{G}(\xi) \in \mathrm{L}^{1}$, i.e., 


$$
\left(1-(2 \pi)^{\mathrm{d}}|\hat{\phi}(\xi)|^{2}\right)|\xi|^{-\mathrm{t}} \in \mathrm{L}^{1},
$$

as desired.

We note that Theorem 3.8 establishes the equivalence of statements (d) and (e) in Theorems 1 and 2 when $t=2 s$.

\section{Proof of Theorems 1 and 2}

With the results of Sections 2 and 3 we can now prove Theorems 1 and 2 :

Proof: The equivalence of (a) and (b) of Theorem 1 is in Theorem 2.9. The implication (c) $\Rightarrow$ (a) is proved in Theorem 2.12, while (b) $\Rightarrow$ (c) is Theorem 2.15. The equivalence of (c) and (c') is easily established. Since the equivalence of (c) and (b) in fact is shown to hold for any given family of wavelets, it follows $\left(\mathrm{c}^{\prime \prime}\right)$ is equivalent to (b). That (d) and $\left(\mathrm{d}^{\prime}\right)$ are equivalent to (a) has been proved in Theorem 3.7. The equivalence of $\left(d^{\prime}\right)$ and $\left(d^{\prime \prime}\right)$ follows from Lemma 3.1, since

$$
\begin{gathered}
\int_{|\xi|<\delta} \sum_{\ell \neq 0}|\hat{\phi}(\xi+2 \pi \ell)|^{2}|\xi|^{-2 s} \mathrm{~d} \xi=\int_{|\xi|<\delta}\left((2 \pi)^{-d}-|\widehat{\phi}(\xi)|^{2}\right)|\xi|^{-2 s} \mathrm{~d} \xi \\
=\int_{|\xi|<\delta}\left((2 \pi)^{-d / 2}-|\widehat{\phi}(\xi)|\right)\left((2 \pi)^{-d / 2}+|\widehat{\phi}(\xi)|\right)|\xi|^{-2 s} \mathrm{~d} \xi .
\end{gathered}
$$

Let us now prove (a) $\Leftrightarrow\left(a^{\prime}\right)$. Clearly we need only show (a) $\Rightarrow\left(a^{\prime}\right)$. This follows easily if we assume the equivalence of $(a)$ and $(b)$ or $(c)$. If we do not assume the existence of a set of wavelets or a scaling function, we can proceed as follows.

Thus assume that the multiresolution approximation yields pointwise order of approximation $\mathrm{s}-\mathrm{d} / 2$ in $\mathrm{H}^{\mathrm{s}}$. Then by the parts of the Theorem already proved, I - P: $\mathrm{H}_{\mathrm{h}}^{\mathrm{s}}$ $\rightarrow \mathrm{L}^{\infty}$ is bounded. To show order of convergence $\mathrm{r}-\mathrm{d} / 2$ in $\mathrm{H}^{\mathrm{r}}$ for $d / 2<\mathrm{r} \leq \mathrm{s}$, we need to show $\mathrm{I}-\mathrm{P}: \mathrm{H}_{\mathrm{h}}^{\mathrm{r}} \rightarrow \mathrm{L}^{\infty}$ is also bounded for such $\mathrm{r}$. The latter follows from decomposing Fourier transforms of functions $\mathrm{f} \in \mathrm{H}_{\mathrm{h}}^{\mathrm{r}}$ as $\hat{\mathrm{f}}=\hat{\mathrm{f}}_{1}+\hat{\mathrm{f}}_{2}$, where $\hat{f}_{1}(\xi)$ is zero for $|\xi| \geq 1$, and $\hat{\mathrm{f}}_{2}(\xi)$ is zero for $|\xi| \leq 1 / 2$. Uniform boundedness of $\mathrm{I}-\mathrm{P}: \mathrm{H}_{\mathrm{h}}^{\mathrm{r}} \rightarrow \mathrm{L}^{\infty}$ on functions of the form $\hat{f_{1}}$ with $\left\|f_{1}\right\|$ h,r $\leq 1$ is easy to show, since for such functions the norm $\|\cdot\|_{h, s}$ is dominated by $\|\cdot\|_{h, r}$. Further, uniform boundedness of I - P: $\mathrm{H}_{\mathrm{h}}^{\mathrm{r}} \rightarrow \mathrm{L}^{\infty}$ on functions of the form $\hat{f}_{2}$ with h,r norm less than 1 also holds, since this class of functions is uniformly bounded in $\mathrm{H}^{\mathrm{r}}$ for $\mathrm{r}>\mathrm{d} / 2$, and so is uniformly bounded in $\mathrm{C}^{0}$. Writing $(\mathrm{I}-\mathrm{P}) \mathrm{f}_{2}=\mathrm{f}_{2}-\mathrm{Pf}_{2}$, clearly the identity is uniformly bounded on $\mathrm{H}^{\mathrm{r}} \rightarrow \mathrm{L}^{\infty}$ for the sub-class of functions $\mathrm{f}_{2}$, and in addition $\mathrm{P}$, having a radially bounded kernel, is also uniformly bounded between the same spaces. The latter follows from the fact that functions of the form $\mathrm{f}_{2}$ with $\mathrm{H}^{\mathrm{r}}$ norm less than 1 are also uniformly bounded in $\mathrm{L}^{\infty}$, so that, since $\mathrm{P}$ has a kernel which is bounded by an $\mathrm{L}^{1}$ convolution kernel, $\mathrm{Pf}_{2}$ for such $\mathrm{f}_{2}$ are uniformly bounded in $\mathrm{L}^{\infty}$. Therefore, it follows that in $\mathrm{H}_{\mathrm{h}}^{\mathrm{r}}$ for $\mathrm{d} / 2<\mathrm{r} \leq \mathrm{s}$, I - P is uniformly bounded, and therefore that we have order of convergence $\mathrm{r}-\mathrm{d} / 2$ in $\mathrm{H}_{\mathrm{h}}^{\mathrm{r}}$ for $\mathrm{r}$ in this range.

To prove that (a) and $\left(\mathrm{a}^{\prime \prime}\right)$ are equivalent it suffices to show that in $\mathrm{H}^{\mathrm{s}}$ for $\mathrm{s}>\mathrm{d} / 2$, the order of approximation, if it is $s-d / 2$, cannot be better than $s-d / 2$. However, if the 
expansion has order of approximation $\mathrm{s}-\mathrm{d} / 2$, then by previously proved parts of the theorem, $\mathrm{E}: \mathrm{H}_{\mathrm{h}}^{\mathrm{s}} \rightarrow \mathrm{L}^{\infty}$ is bounded, so by Proposition 2.8 the best order of approximation in $\mathrm{H}_{\mathrm{h}}^{\mathrm{s}}$ is $\mathrm{s}-\mathrm{d} / 2$. That $\left(\mathrm{a}^{\prime \prime \prime}\right)$ follows from $\left(\mathrm{a}^{\prime \prime}\right)$ can be shown by an argument identical to the above proof that $\left(\mathrm{a}^{\prime \prime}\right)$ follows from $(\mathrm{a})$.

Finally the equivalence of $(e)$ of Theorem 2 with $(d)$ of Theorem 1 follows from Theorem 3.8, and the equivalence of $\left(\mathrm{d}^{\prime \prime}\right)$ with $\left(e^{\prime}\right)$ of Theorem 2 is apparent. The equivalence of $\left(e^{\prime}\right)$ and $\left(e^{\prime \prime}\right)$ in Theorem 2 follows from (3.9), in the form

$$
1-\left|\mathrm{m}_{0}(\xi)\right|^{2}=\sum_{\epsilon \neq 0}\left|\mathrm{~m}_{0}(\xi+\pi \epsilon)\right|^{2},
$$

together with a change of variable in the integral of $\left(e^{\prime \prime}\right)$.

This completes the proofs of the two theorems.

\section{Arbitrarily slow convergence}

We show here that there exist functions whose wavelet expansions converge arbitrarily slowly.

Consider in one dimension $f(x)=\left\{\begin{array}{c}x^{\epsilon} \text { for }|x|<1 \\ 0 \text { for }|x|>2\end{array}\right.$, and which is smooth everywhere. Recall

$$
P_{n} f(x)=\int P_{n}(x, y) f(y) d y=2^{n} \int P_{0}\left(2^{n} x, 2^{n} y\right) f(y) d y=\int P_{0}\left(2^{n} x, y\right) f\left(2^{-n} y\right) d y .
$$

so that at $x=0$,

$$
\begin{aligned}
& E_{n} f(0) \equiv\left(I-P_{n}\right) f(0) \\
& =P_{n} f(0) \\
& =\int \mathrm{P}_{0}(0, \mathrm{y}) \mathrm{f}\left(2^{-\mathrm{n}} \mathrm{y}\right) \mathrm{dy} \\
& =\left(\int_{\mathrm{I}_{\mathrm{n}}}+\int_{\mathrm{J}_{\mathrm{n}}}\right) \mathrm{P}_{0}(0, \mathrm{y}) \mathrm{f}\left(2^{-\mathrm{n}} \mathrm{y}\right) \mathrm{dy},
\end{aligned}
$$

where $I_{n}=\left(-2^{n}, 2^{n}\right)$ and $J_{n}=\left(2^{n}, \infty\right) \cup\left(-2^{n}, \infty\right)$. Now

$\int_{I_{n}} P_{0}(0, y) f\left(2^{-n} y\right) d y=\int_{-2^{n}}^{2^{n}} P_{0}(0, y)\left(2^{-n} y\right)^{\epsilon} d y=2^{-n \epsilon} \int_{-2^{n}}^{2^{n}} P_{0}(0, y) y^{\epsilon} d y$

For simplicity we assume the scaling function $\phi$ has compact support, so the same is true of $\mathrm{P}(0, \mathrm{y})$. Thus the integral over $\mathrm{I}_{n}$ will be $\mathrm{O}\left(2^{-\mathrm{n} \epsilon}\right)$. Since $P_{n}(0, y)$ has compact support, the integral over $J_{n}$ eventually vanishes, and we have error at the point 0 given by $O\left(2^{-n \epsilon}\right)$. Since $\epsilon$ can be arbitrarily small, convergence can be arbitrarily slow.

As a final remark we note that because the scaling relation between $E_{n-1}$ and $E_{n}$ is the same as that between $E_{n}$ and $E_{n+1}$, convergence must be exponential, i.e., $\left\|E_{n}\right\|_{A \rightarrow L^{\infty}}$ vanishes exponentially in $n$, whenever the Banach space $A$ has a norm with the 
homogeneous space property $\|f(c x)\| \sim c^{\alpha}\|f(x)\|(c \rightarrow \infty)$, which is the case with almost all spaces currently of interest.

Acknowledgments: The first author thanks the U.S. Fulbright Commission and the University of Warsaw for its support, and the second author acknowledges the Courant Institute of Mathematical Sciences for a stimulating year. We wish to thank several readers for reading and corrections of this manuscript, and in particular to one to whom we are indebted for some alternative and better proofs, some of which we have adopted essentially unchanged in this paper.

\section{REFERENCES:}

[Au] P. Auscher, Wavelet bases for $\mathrm{L}^{2}(\mathbb{R})$ with rational dilation factor, in Ruskai. et. al., eds., Wavelets and Their Applications, Jones and Bartlett, Boston, 1992, 439-452.

[BM] M. Buhmann, and C. Micchelli, Spline prewavelets for non-uniform knots, Numerische Mathematik 61 (1992), 455-474.

[C] L. Carleson, On convergence and growth of partial sums of Fourier series, Acta Mathematica 116 (1966), 135-195.

[D1] I. Daubechies, Orthonormal bases of compactly supported wavelets, Comm. Pure Appl. Math. 41 (1988), 909-996.

[D2] I. Daubechies, Ten Lectures on Wavelets, CBMS-NSF Series in Applied Mathematics, SIAM, 1992.

[D3] I. Daubechies, Lecture Notes, CBMS Conference, Univ. Mass. at Lowell, 1990.

[BR] deBoor C. and A. Ron, Fourier analysis of the approximation power of principal shift-invariant spaces, Constr. Approx. 8 (1992), 427-462.

[BDR] deBoor, C., R. DeVore, and A. Ron, Approximation from shift-invariant subspaces of $\mathrm{L}^{2}\left(\mathbb{R}^{\mathrm{d}}\right)$, Trans. Amer. Math. Soc. 341 (1994), 787-806.

[F] C. Fefferman, The new multiplier theorem for the ball, Ann. Math. 94 (1971), 330-336.

[Gr] K. Grochenig, Analyse multiéchelle et bases d'ondelettes, CRAS Paris, Série I (1987), 13-17.

[GK1] David Gurarie and Mark A. Kon, Radial bounds for perturbations of elliptic operators, J. Functional Analysis 56 (1984), 99-123. 
[GK2] David Gurarie and Mark A. Kon, Resolvents and regularity properties of elliptic operators, in Operator Theory: Advances and Applications, C. Apostol, Ed., Birkhauser Verlag, 1983.

[HT] R. Holschneider and Ph. Tchamitchian, Régularité locale de la fonction `nondifferentiable' de Riemann, in Les Ondelettes in 1989, P. G. LeMarie, ed., Springer-Verlag, Berlin, 1990.

[JZ] K. Jetter and D. X. Zhou, Order of linear approximation in shift-invariant spaces, Constr. Approx. 11 (1995), 423-438.

[KKR1] S. Kelly, M. Kon, and L. Raphael, Local convergence of wavelet expansions, J. Functional Analysis 126 (1994), 102-138.

[KKR2] S. Kelly, M. Kon, and L. Raphael, Pointwise convergence of wavelet expansions, Bull. Amer. Math Soc. 30 (1994), 87-94.

[KR1] M. Kon and L. Raphael, New multiplier methods for summing classical eigenfunction expansions, J. Differential Equations 50 (1983), 391-406.

[KR2] M. Kon and L. Raphael, Characterizing convergence rates for multiresolution approximations, in Signal and Image Representation in Combined Spaces, J. Zeevi and Ronald Coifman, Eds., Academic Press, 1998, 415-437.

[Ma] S. Mallat, Multiresolution approximation and wavelets, Trans. Am. Math. Soc. 315 (1989), 69-88.

[Me] Y. Meyer, Ondelettes, Hermann, Paris, 1990.

[R] M.B. Ruskai, G. Beylkin, R. Coifman, I. Daubechies, S. Mallat, Y. Meyer, and L. Raphael, eds., Wavelets and their applications, Jones and Bartlett, Boston, 1992.

[SW] Eli Stein and Guido Weiss, Introduction to Fourier Analysis on Euclidean Spaces, Princeton University Press, Princeton, 1971.

[Ste] E. Stein, Singular Integrals and Differentiability Properties of Functions, Princeton University Press, Princeton, 1970.

[Str] G. Strang, Wavelets and dilation equations, a brief introduction, SIAM Review $\mathbf{3 1}$ (1989), 614-627.

[SF] G. Strang, and G. Fix, A Fourier analysis of the finite element variational method, in Constructive Aspects of Functional Analysis, Edizioni Cremonese, Rome, 1973. 
[St] R. Strichartz, Wavelet expansions of fractal measures, J. Geometric Anal. 1 (1991), 269-289.

[SP] W. Sweldens and R. Piessens, Asymptotic error expansions for wavelet approximations of smooth functions, Technical Report TW164, Katholieke Universiteit Leuben, 1992.

[Wa] G. Walter, Approximation of the delta function by wavelets, J. Approx. Theory 71 (1992), 329-343

[Wo] P. Wojtaszczyk, A Mathematical Introduction to Wavelets, Cambridge University Press, Cambridge, 1997. 